\title{
Sex differences in the response to emotional distraction: an event-related fMRI investigation
}

\author{
Alexandru D. Iordan • Sanda Dolcos • \\ Ekaterina Denkova $\cdot$ Florin Dolcos
}

Published online: 6 January 2013

(C) Psychonomic Society, Inc. 2013

\begin{abstract}
Evidence has suggested that women have greater emotional reactivity than men. However, it is unclear whether these differences in basic emotional responses are also associated with differences in emotional distractibility, and what the neural mechanisms that implement differences in emotional distractibility between women and men are. Functional MRI recording was used in conjunction with a working memory (WM) task, with emotional distraction (angry faces) presented during the interval between the memoranda and the probes. First, we found an increased impact of emotional distraction among women in trials associated with high-confidence responses, in the context of overall similar WM performance in women and men. Second, women showed increased sensitivity to emotional distraction in brain areas associated with "hot" emotional processing, whereas men showed increased sensitivity in areas associated with "cold" executive processing, in the context of overall similar patterns of response to emotional distraction in women and men. Third, a sex-related dorsalventral hemispheric dissociation emerged in the lateral PFC related to coping with emotional distraction, with women showing a positive correlation with WM performance in left ventral PFC, and men showing similar effects in the right dorsal PFC. In addition to extending to men results that have previously been reported in women, by showing that both
\end{abstract}

A.D.I. and S.D. share first-authorship for this article.

A. D. Iordan $\cdot$ S. Dolcos $\cdot$ F. Dolcos

University of Illinois at Urbana-Champaign, Urbana, IL, USA

E. Denkova

University of Alberta, Edmonton, Alberta, Canada

F. Dolcos $(\triangle)$

Beckman Institute for Advanced Science \& Technology,

University of Illinois at Urbana-Champaign,

405 North Mathews Avenue,

Urbana, IL 61801, USA

e-mail: fdolcos@illinois.edu sexes engage mechanisms that are similar overall in response to emotional distraction, the present study identifies sex differences in both the response to and coping with emotional distraction. These results have implications for understanding sex differences in the susceptibility to affective disorders, in which basic emotional responses, emotional distractibility, and coping abilities are altered.

Keywords Emotional interference $\cdot$ Social anxiety · Amygdala $\cdot$ Subgenual anterior cingulate cortex (sgACC) . Dorsolateral/ventrolateral prefrontal cortex (dlPFC/vlPFC) . Fusiform face area (FFA)

Probably due to their enhanced relevance for survival, emotional stimuli tend to capture our attention more easily than do neutral ones, and thus they can be powerful distractors, particularly if they are task-irrelevant. Because people vary in their responses to and ability to cope with emotional distraction, goal-irrelevant emotions may impact them differently. The present study focused on sex differences in the response to emotional distraction. Although the available anecdotal and scientific evidence suggests that women and men may respond differently to emotional situations, it is still unclear whether differences in emotional reactivity are also associated with differences in emotional distractibility, and whether men and women engage similar or dissociable mechanisms in their responses to emotional distraction. Previous studies involving dual-task paradigms with emotional distraction that have allowed for clear dissociations of the time courses of response in the ventral-affective and dorsal-cognitive brain systems have included only female subjects (Denkova, Wong, Dolcos, Sung, Wang, Coupland, \& Dolcos, 2010; Dolcos, Diaz-Granados, Wang, \& McCarthy, 2008; Dolcos, Kragel, Wang, \& McCarthy, 2006; Dolcos \& McCarthy, 2006). Hence, it is not clear whether the observed effects could also be extended to males. For the present investigation, we used functional 
magnetic resonance imaging (fMRI) in conjunction with behavioral measures to investigate how emotional distraction impairs cognition in women and men, as well as the neural mechanisms associated with these effects. The main questions concerned the link between emotional reactivity and emotional distraction-specifically, whether the enhanced emotional reactivity observed in female subjects is linked to enhanced emotional distractibility - and identifying the neural mechanisms that implement differences in emotional distractibility between women and men. Investigation of these issues in nonclinical individuals has potential implications for a better understanding of these phenomena as independent or correlated factors contributing to differential vulnerability to affective disorders in women and men.

Behavioral and brain-imaging evidence has suggested that enhanced emotional reactivity in women may also lead to enhanced emotional distractibility. The available evidence has shown that in addition to enhanced emotional competence (Barrett, Lane, Sechrest, \& Schwartz, 2000; Kring \& Gordon, 1998; Seidlitz \& Diener, 1998), women also show enhanced reactivity to emotional challenge (Hamann \& Canli, 2004; Lang, Greenwald, Bradley, \& Hamm, 1993; Shields, 1991), specificity in the deployment of emotion regulation strategies (Denkova, Dolcos, \& Dolcos, 2012; Domes et al., 2010; Mak, Hu, Zhang, Xiao, \& Lee, 2009; Matud, 2004; McRae, Ochsner, Mauss, Gabrieli, \& Gross, 2008; Thayer, Rossy, Ruiz-Padial, \& Johnsen, 2003), and increased susceptibility to affective disorders (i.e., a lifetime prevalence of mood and anxiety disorders nearly two times higher than that of men; Bekker \& van Mens-Verhulst, 2007; Kessler, 2003). Evidence from brain-imaging studies points to sex-related differences, primarily in the brain regions associated with emotion processing, such as the amygdala (AMY) and the orbitofrontal cortex (OFC) (Goldstein et al., 2001; Gur, Gunning-Dixon, Bilker, \& Gur, 2002), but differences in brain regions associated with the cognitive control of emotion, such as the prefrontal cortex (PFC) (Koch et al., 2007; McRae et al., 2008), have also been identified. This evidence raises the possibility that the same mechanisms that help generate the enhanced emotional experience in women could also be partially responsible for enhanced sensitivity to emotional factors. However, in these previous investigations, the emotional stimuli have been taskrelevant, and thus it is not known whether similar effects are produced when the emotional information is presented as taskirrelevant distraction.

Brain-imaging studies in which emotional information has been presented as transient distraction have proved informative in identifying the neural circuitry related to the impact of emotional distraction on ongoing cognitive processes, as well as the neural correlates of coping with distracting emotions (reviewed in Dolcos, Iordan, \& Dolcos, 2011). In a series of studies by Dolcos and colleagues, the neural correlates that mediate emotion-cognition interactions were investigated using a paradigm in which emotional task-irrelevant distractors were presented during the delay interval of a working memory (WM) task (Dolcos et al., 2008; Dolcos et al., 2006; Dolcos \& McCarthy, 2006; Dolcos, Miller, Kragel, Jha, \& McCarthy, 2007). The main finding of these studies was that the impairing effect of emotional distraction was linked to opposing patterns of activity in ventral brain regions associated with "hot" emotion processing (the HotEmo system) and dorsal regions associated with "cold" executive processing (the ColdEx system). Specifically, emotional distractors enhanced activity in emotion-processing regions such as AMY, the ventrolateral prefrontal cortex (vlPFC), and medial PFC, while disrupting delay activity in dorsal executive brain regions such as the dorsolateral PFC (dlPFC) and the lateral parietal cortex (LPC). Given the role of the latter brain regions in attentional processes and active maintenance of goalrelevant information in WM (D'Esposito, Postle, \& Rypma, 2000; Hopfinger, Buonocore, \& Mangun, 2000; Levy \& Goldman-Rakic, 2000; Miller \& Cohen, 2001), these findings suggest that activity in the affective and executive neural systems is strongly interconnected, such that increased activity in the ventral affective regions disrupts activity in the dorsal system and results in cognitive impairment.

Evidence from follow-up investigations (Denkova et al., 2010) showed that these opposite patterns of activity in the ventral and dorsal neural systems in the presence of emotional distraction that induces general negative affect (pictures from the International Affective Picture System; Lang, Bradley, \& Cuthbert, 2008) were also observed with stimuli that induced specific emotions (i.e., angry faces) in female subjects. Employing an adapted version of the WM task with distraction used by Dolcos and McCarthy (2006), the study of Denkova et al. (2010) provided support for the idea that the impact of angry faces used as distractors is mainly linked to bottom-up effects. These effects were reflected in enhanced activity in brain regions associated with visual and emotional processing (fusiform gyrus [FG] and AMY) and in regions associated with self-referential processing and experiencing of emotion (ventromedial PFC [vmPFC]), which diverts attention from the main WM task and leads to impaired performance.

The vmPFC is part of the so-called default-mode network, which shares common regions with the ventral affective network. Anatomically, the default-mode network comprises mainly midline cortical structures, such as the medial PFC and the posterior parietal cortex (PPC), along with lateral parietal (LPC) and superior temporal cortex (STC) (Buckner, Andrews-Hanna, \& Schacter, 2008; Raichle et al., 2001). The default-mode network is involved in self-referential processing (Northoff \& Bermpohl, 2004; Northoff et al., 2006), including focus on autobiographical memories (Svoboda, McKinnon, \& Levine, 2006), planning for the future 
(D’Argembeau et al., 2010; Schacter, Addis, \& Buckner, 2008), and evaluating the salience of internal and external cues (Phan et al., 2004). In addition, previous evidence has suggested that the default-mode network is also involved in emotion processing (Harrison et al., 2008; Pitroda, Angstadt, McCloskey, Coccaro, \& Phan, 2008; Wiebking et al., 2011) and in affective decision making (AndrewsHanna, Reidler, Sepulcre, Poulin, \& Buckner, 2010; Ochsner et al., 2004). Typically, the default-mode network shows increased activity during rest and displays deactivation from its resting state in response to external attentiondemanding tasks (Greicius, Krasnow, Reiss, \& Menon, 2003; Raichle et al., 2001). While the evidence suggests that default-mode network resting state activity does not differ between women and men (Weissman-Fogel, Moayedi, Taylor, Pope, \& Davis, 2010), it is possible that areas of the default-mode network are differentially engaged in the context of transient emotional distraction.

The study by Denkova et al. (2010) identified changes in the HotEmo and ColdEx systems and in areas of the defaultmode network (i.e., vmPFC) in response to emotional distraction, but this study involved only female subjects. Hence, it is not clear whether similar effects would also be observed in males, and whether women and men differ both in the detrimental impact of emotional distraction and in the engagement of the cognitive control mechanisms needed to cope with the presence of emotional distraction. Therefore, the main goals of the present study were to determine whether sex differences in basic emotional reactivity are associated with differences in emotional distractibility, and to identify the neural mechanisms that implement differences in emotional distractibility between women and men. Brain activity was recorded using event-related fMRI while healthy male and female subjects performed a WM task with emotional distraction. The behavioral assessments involved measures of WM performance and subjective ratings of the distractors, along with measures of posttask affective states. To further investigate the significance of possible differences in behavior and brain activity, brainbehavior relationships were also investigated by calculating covariations between the brain responses to transient emotional distraction and WM performance.

From the extant evidence, we made the following three predictions: First, regarding behavioral performance, consistent with evidence of enhanced response in women when emotional information helps cognition (i.e., enhanced memory for emotional events; Canli, Desmond, Zhao, \& Gabrieli, 2002), we expected that women would also be more susceptible to the detrimental impact of emotional information, presented as task-irrelevant distraction concurrent with a main cognitive task. Second, regarding the brain-imaging results, we expected that sex-related differences in the behavioral impact of emotional distraction would be linked to differences in both brain regions whose engagement reflects a detrimental impact of emotional distraction and brain regions engaged to cope with the presence of emotional distraction. Third, regarding the brain-behavior relationships, we expected that negative covariations between brain activity and WM performance would presumably be indicative of mechanisms whose engagement leads to detrimental effects of emotional distraction on performance, whereas positive covariations would be indicative of mechanisms engaged to counteract the negative impact of emotional distraction.

\section{Method}

Subjects

A group of 36 healthy, young, right-handed adults (18-33 years of age, average $=22.86, S D=3.89 ; 18$ women, 18 men) participated in the study; the female sample overlapped with that of Denkova et al. (2010). The data from two female and two male subjects were excluded from the analyses because of incompleteness (e.g., due to missing runs). Hence, the analyses reported here are based on data from 32 subjects (average age $=$ 23.12, $S D=4.04 ; 16$ women, 16 men). The women and men did not differ in age $[t(30)=0.61, p>.5]$. The level-ofconfidence data from two male subjects and emotionalratings data from one female subject were not recorded due to technical problems, and hence could not be included in the corresponding analyses. The experimental protocol was approved by the Health Research Ethics Board at the University of Alberta, and all subjects provided written informed consent.

\section{Stimuli}

The subjects performed a delayed-response WM task for faces with emotional distraction, which had been used on only female subjects in our previous study (Denkova et al., 2010; Fig. 1). This is a modified version of our delayedresponse WM task with distraction from Dolcos and McCarthy (2006), adapted to be used with angry-face distractors. The memoranda consisted of sets of three human faces (50\% female, $50 \%$ male; Chuah et al., 2010; Dolcos et al., 2008; Dolcos \& McCarthy, 2006), chosen to maximize similarities, for increased task difficulty. The distractors, selected from the NimStim Face Stimulus Set (www.macbrain.org/resources.htm; Tottenham, Borscheid, Ellertsen, Marcus, \& Nelson, 2002), were presented during the delay interval between the memoranda and probes, and consisted of morphed angry faces, neutral faces, and scrambled faces $(50 \%$ female, $50 \%$ male). Morphed facial stimuli were used in order to induce responses closer to those of real-life social interactions; the morphing was performed using Winmorph (www.debugmode.com/ winmorph/). The scrambled faces had the same average 


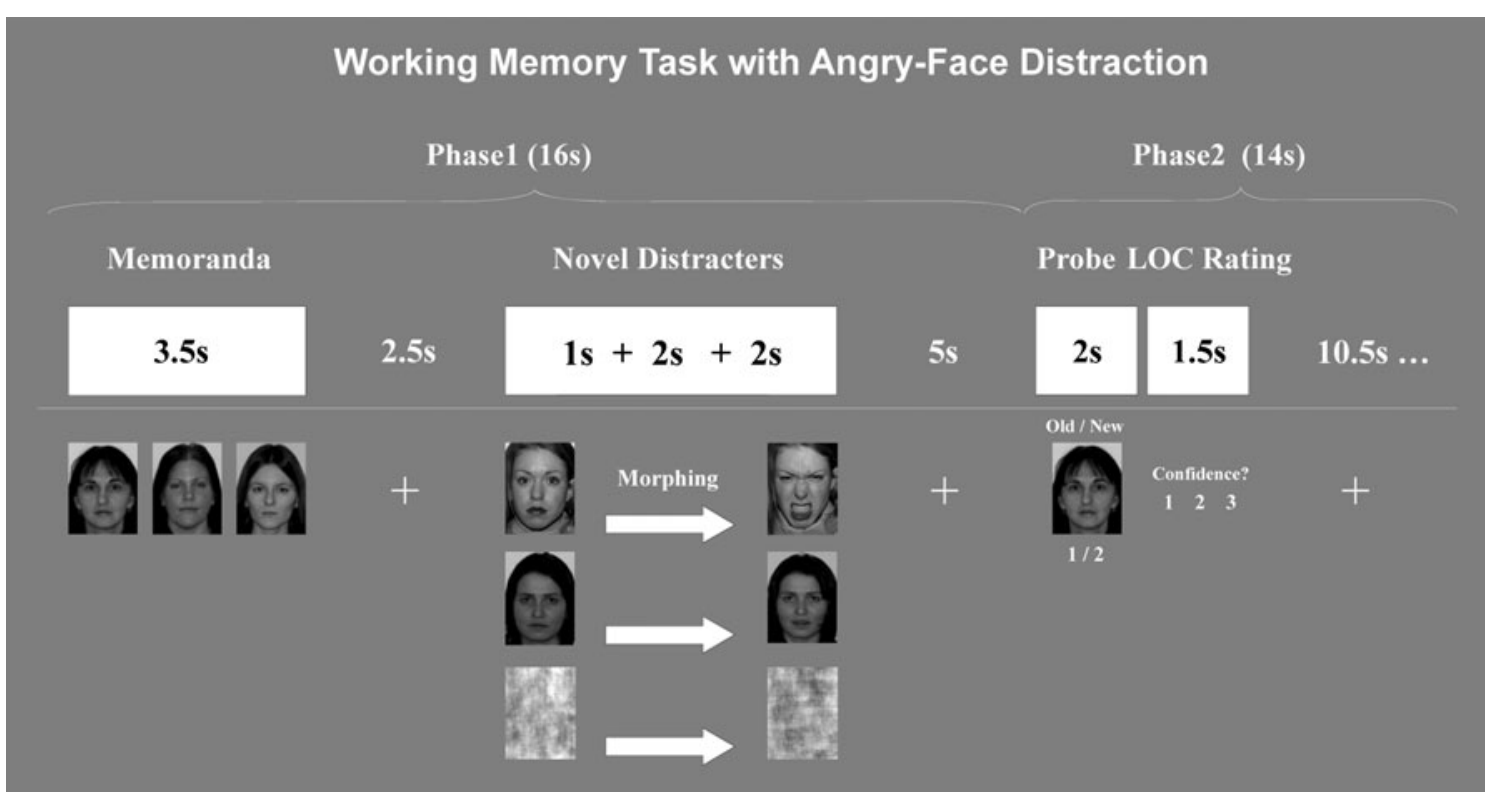

Fig. 1 Diagram of the WM task with angry-face distraction. Functional magnetic resonance imaging (fMRI) data were recorded while subjects performed a working memory (WM) task with distraction. To increase the impact of distraction, the novel distractors were morphed. WM performance was measured using an old/new recognition memory task, followed by a level-of-confidence (LOC) task $(1=$ low, $2=$ medium, 3 =high). Written informed consent was obtained for

spatial frequency and luminance as the meaningful angry and neutral faces, and served as no-distraction perceptual controls. A total of 90 experimental trials, identified on the basis of types of distractors, were included, as follows: 30 with angry faces, 30 with neutral faces, and 30 with scrambled faces. All of the stimuli were presented in color.

\section{Experimental procedures}

Six sets of 15 trials each were created (five angry, five neutral, and five scrambled faces per set) and randomly assigned to six experimental blocks/runs. To avoid induction of longerlasting effects, the trials within each block were pseudorandomized, so that no more than two consecutive trials of the same type were presented. To prevent possible biases that could result from using the same run order, the subjects were assigned different run orders; a total of six different run orders were involved. Each trial started with the presentation of face memoranda (3.5 s), which subjects were instructed to encode and maintain in WM during the delay interval between the offset of the memoranda and the onset of the memory probe $(12.5 \mathrm{~s})$. Presentation of novel distractors started $2.5 \mathrm{~s}$ after the offset of the memoranda and occurred for a total time of $5 \mathrm{~s}$. All of the distractors started as static stimuli (either neutral or scrambled), and then after a short delay (1 s), they morphed for a 2-s period, which was followed by another static presentation of the final morphed faces $(2 \mathrm{~s})$. Half of the initially neutral faces morphed into angry faces, and the other half photograph publication of all faces illustrated in the figure that are not part of the standardized NimStim Face Stimulus Set. From "The Impact of Anxiety-Inducing Distraction on Cognitive Performance: A Combined Brain Imaging and Personality Investigation," by E. Denkova, G. Wong, S. Dolcos, K. Sung, L. Wang, N. Coupland, and F. Dolcos, 2010, PLoS ONE, 5, e14150, p. 3. Copyright 2010 by Denkova et al. Adapted with permission

morphed into neutral faces. Also, to control for motion-related changes, half of the scrambled faces morphed into the corresponding scrambled angry faces, and the other half morphed into the corresponding scrambled neutral faces.

The subjects were instructed to look at the distractors but to maintain focus on the WM task, and when a single face probe appeared, they were to indicate by a buttonpress whether the face was part of the current memorandum (old) or not (new); $50 \%$ of the probes were old and $50 \%$ were new. The subjects were instructed to make quick and accurate responses while the probes were on the screen, and then they also rated the level of confidence (LOC) of their responses, using a three-point Likert scale $(1=$ lowest, $3=$ highest). The LOC rating was followed by a 10.5 -s intertrial interval, so as to allow the hemodynamic response to return to baseline. The total length of each trial was $30 \mathrm{~s}$. Following scanning, subjects performed an emotionalrating task, in which they had to rate how angry they perceived the emotional and neutral distractors to be, using a nine-point Likert scale $(1=$ not angry at all, $9=$ very angry). These ratings were assessed to confirm that the angry faces were perceived as being more emotional than the neutral faces, and to investigate possible sex-related differences in the ratings. Finally, to assess changes in emotional states as a result of participating in the study, subjects completed the State scales of the Positive and Negative Affective Schedule (PANAS-S; Watson, Clark, \& 
Tellegen, 1988) and the State-Trait Anxiety Inventory (STAI-S; Spielberger, Gorsuch, \& Lushene, 1970), at both the beginning and the end of the study.

\section{Imaging protocol}

Scanning was conducted on a 1.5-T Siemens Sonata scanner. After the sagittal localizer and the 3-D MPRAGE anatomical images $(\mathrm{TR}=1,600 \mathrm{~ms} ; \mathrm{TE}=3.82 \mathrm{~ms} ; \mathrm{FOV}=256 \times 256 \mathrm{~mm}$; volume size $=112$ slices; voxel size $=1 \times 1 \times 1 \mathrm{~mm}^{3}$ ), six blocks of full-brain EPI functional images were acquired axially $(\mathrm{TR}=2,000 \mathrm{~ms} ; \mathrm{TE}=40 \mathrm{~ms} ; \mathrm{FOV}=256 \times$ $256 \mathrm{~mm}$; volume size $=28$ slices; voxel size $=4 \times 4 \times 4 \mathrm{~mm}^{3}$ ).

\section{Behavioral data analyses}

Responses in the WM task were classified into one of the four categories derived from signal detection theory (Macmillan \& Creelman, 1991): (1) hits = probes from memoranda (old) correctly classified as "old"; (2) misses = probes from memoranda incorrectly classified as "new"; (3) correct rejections $(\mathrm{CRs})=$ new probes correctly classified as "new"; and (4) false alarms (FAs) = new faces incorrectly classified as "old." Corrected recognition scores (\% Hits - \% FAs) were also calculated for each subject. Differences in WM performance among the three trial types (emotional vs. neutral vs. scrambled) and between males and females were assessed using analyses of variance (ANOVAs) and $t$ statistics; sex-related differences in emotional ratings and affective state were also assessed.

\section{fMRI data analyses}

Statistical analyses were preceded by the following preprocessing steps (performed with SPM2-Statistical Parametric Mapping): TR alignment, motion correction, coregistration, normalization, and smoothing $\left(8-\mathrm{mm}^{3} \mathrm{kernel}\right)$. For the data analysis, we used in-house custom MATLAB scripts involving both whole-brain voxel-wise and region-of-interest (ROI) analyses (Dolcos \& McCarthy, 2006) to compare the brain activity associated with the conditions of interest (e.g., trials with angry vs. trials with neutral distractors). For subject-level analyses, the fMRI signal was selectively averaged in each subject's data as a function of trial type (i.e., angry, neutral, or scrambled distractors) and time point (one pre- and 13 post-stimulus-onset time points) using custom MATLAB software, and pair-wise $t$ statistics for the contrast of interest were calculated for each subject; no assumption was made about the shape of the hemodynamic response function (Dolcos et al., 2008; Dolcos \& McCarthy, 2006; Morey et al., 2009). The individual analyses produced whole-brain average and activation $t$ maps for each condition, contrast of interest, and TR/time point. The outputs of the subject-level analyses were used as inputs for second- level, random-effects within-group and across-groups analyses. The analyses focused on any effects observed at time points within the 14- to 16 -s period after the memoranda onset (TR 9), when the differential effects of the distractors were most evident (Denkova et al., 2010; Dolcos \& McCarthy, 2006); exceptions are noted in the tables as necessary. Conjunction analyses involved masking procedures performed in MATLAB using the logical function AND; thus, only voxels that met the threshold criteria in each of the contributing $t$ maps survived the masking procedure. This procedure is consistent with the conjunction null hypothesis testing (Nichols, Brett, Andersson, Wager, $\&$ Poline, 2005). A joint threshold of $p<.0005$ was used in all of the analyses, and an extent threshold of 10 contiguous voxels was used in each of the contributing maps (Lieberman \& Cunningham, 2009).

The main goals of the present study were to determine whether sex differences in basic emotional reactivity are associated with differences in emotional distractibility and to identify the neural mechanisms that implement differences in emotional distractibility between women and men. To accomplish this goal, brain regions in the HotEmo and ColdEx systems were first defined as a priori functional ROIs, on the basis of findings from the original study by Dolcos and McCarthy (2006), who used a similar WM task with highly arousing negative pictures as novel distractors. For the purposes of the present study, the following two $t$ maps identifying brain areas showing general sensitivity to negative distraction were used: Emo $>$ Scr, to identify regions of the ventral HotEmo system, and Scr $>$ Emo, to identify regions of the dorsal ColdEx system. The functional ROI masks were calculated by merging suprathreshold brain activity in the window from 12 to $18 \mathrm{~s}$ (TRs 8 - 10) after memoranda onset, which corresponds to the time interval in which the peak effects reported by Dolcos and McCarthy had occurred. This confined the identification of brain areas showing specific responses to angry-face distractors, investigated here, to the brain areas showing general sensitivity to negative distraction that had been investigated by Dolcos and McCarthy. A threshold of $p<.05$ (uncorrected) was used for each of these individual masks. Then, two complementary approaches were used to identify areas showing specific sensitivity to the angry-face distractors. The first set of analyses focused on the overall response to angry-face distraction, in order to identify brain areas that are common to women and men in the response to emotional distraction, independent of WM performance. These analyses were performed on the fMRI data for all trials (i.e., 30 per condition), as it was previously reported in the data from female subjects (Denkova et al., 2010). A second set of analyses then focused on the specific impact of angry-face distraction on WM performance, to identify brain activity linked to differences in WM performance between emotional and neutral trials and between male 
and female subjects. These analyses were performed on the fMRI data from trials in which sex differences were identified in WM performance (i.e., LOC 3). Hits and CRs were collapsed together in all analyses, which are described in detail below.

Identification of common brain activity in response to emotional distraction in women and men To identify the brain regions whose activity was sensitive to the presence of angryface distractors in both women and men, an analysis consisting of the following two steps was performed (Denkova et al., 2010). First, the ventral and dorsal brain regions sensitive to angry-face distractors were separately identified in women and men: that is, $($ Emo $>$ Scr $) \cap($ Emo $>$ Neu $)$, for the ventral system, and $($ Emo $<\mathrm{Scr}) \cap(\mathrm{Emo}<\mathrm{Neu})$, for the dorsal system. The joint threshold of the resulting conjunction maps was $p<.0005$ (Fisher, 1950), resulting from multiplying the $p$ values of the contributing maps: that is, $p<.01$ for the most dissimilar conditions (Emo $>$ Scr and Emo $<$ Scr), and $p<.05$ for contrasts between more similar conditions (Emo $>\mathrm{Neu}$ and Emo $<\mathrm{Neu}$ ). This approach also maintained consistency with our previous investigation in women only (Denkova et al., 2010) and allowed for drawing comparable conclusions. Second, conjunction maps identifying the overlapping brain regions sensitive to angry-face distraction in both men and women were calculated for the HotEmo and ColdEx systems: that is, [women $($ Emo $>$ Scr $) \cap($ Emo $>$ Neu $)] \cap[$ men $($ Emo $>$ $\mathrm{Scr}) \cap(\mathrm{Emo}>\mathrm{Neu})$ ], for the HotEmo system, and [women $($ Emo $<$ Scr $) \cap($ Emo $<$ Neu $)] \cap[$ men $($ Emo $<$ Scr $) \cap($ Emo $<$ $\mathrm{Neu})]$, for the ColdEx system.

Identification of brain activity linked to sex differences in WM performance To identify regions of the ventral and dorsal systems showing sex-related differential patterns of responses linked to WM performance, an analysis was performed only for the correct LOC 3 trials in which an emotion effect had been observed in women (Denkova et al., 2010) and sex-related differences in performance were identified. This second approach used a different set of contrasts than had the first, in order to ensure that the investigation was not limited to areas showing similar effects in women and men. The analysis consisted of the following two steps. First, a between-groups analysis comparing the responses to emotional distraction between females and males was performed using a two-sample $t$ test; for this test, the subject-level average signal for the emotional LOC 3 trials was used as the input for second-level betweengroups $t$ tests. Second, to make sure that the regions identified by the between-groups analysis were also sensitive to the effect of emotional distraction, the resulting $t$ maps were inclusively masked with statistical maps identifying a main effect of emotion relative to baseline (i.e., increased vs. decreased activity in the ventral or dorsal system, respectively). To identify brain areas showing increased response to emotional distraction in women versus men, the following conjunction maps were used: $(\mathrm{EmoF}>$ EmoM $) \cap($ EmoF $>$ Baseline $)$, for activity in the ventral system, and $(\mathrm{EmoF}>\mathrm{EmoM}) \cap(\mathrm{EmoM}<$ baseline $)$, for activity in the dorsal system. Similarly, to identify patterns showing increased response to emotional distraction in men versus women, the following conjunction maps were used: $($ EmoM $>$ EmoF $) \cap($ EmoM $>$ baseline $)$, for activity in the ventral system, and $(\mathrm{EmoM}>\mathrm{EmoF}) \cap(\mathrm{EmoF}<$ Baseline $)$, for activity in the dorsal system. The joint threshold of the resulting conjunction maps was $p<.0005$ (Fisher, 1950), resulting from multiplying the $p$ values of the contributing maps $(.05 \times .01)$.

Identification of brain-behavior relationships To further investigate the significance of differences in brain activation, brain-behavior relationships were also investigated by calculating covariations between the fMRI signals in response to transient angry-face distraction and WM performance. These analyses identified brain regions whose activity was sensitive to individual variations in WM performance and was specific to emotional distraction. Negative brain-behavior covariations were expected to be indicative of processing leading to detrimental effects on WM performance, whereas positive covariations were presumed to be indicative of processing engaged to cope with distraction. These analyses involved the following three strategies: (1) investigation in men of covariations of brain activity with WM performance in the same regions that had been identified in women (Denkova et al., 2010); (2) separate investigation in women and men of covariations of activity in brain regions where sex differences in activation linked to differences in WM performance had been identified by the analyses described in the previous paragraph; and (3) exploratory analyses, in both women and men, to identify covariations between brain activity and WM performance in brain areas showing sensitivity to emotional distraction (increased or decreased activity), in the absence of sex differences in activation in the LOC 3 responses, as it is possible that differences in correlations could be observed in the absence of differences in activation. For the latter two strategies, the conjunction analyses performed to investigate brain-behavior relationships consisted of the following two steps. First, correlation maps identifying covariations between brain activity in the presence of distraction and scores indexing WM performance (i.e., corrected recognition scores $=\%$ Hits $-\%$ False Alarms) were calculated. Then, these correlation maps were inclusively masked with maps identifying a main effect of emotion (increased or decreased) relative to the baseline. The joint threshold of the conjunction map was $p<.0005$ (Fisher, 1950), resulting from multiplying 
the $p$ values of the contributing maps obtained independently $(.05 \times .01)$.

Confirmatory ROI investigations Finally, activity in the main brain regions identified by the whole-brain voxel-wise analyses was subjected to further confirmatory investigations, using a functional ROI approach. This involved extraction of the fMRI signal, for each subject, condition, and time point, from voxels identified by the group-level conjunction analyses. Then, across-subjects averages of the extracted MR signal (expressed as percentages of signal change) recorded at the delay peak time point (i.e., 14-16 s following memoranda onset) and correlations between the extracted fMRI signal and the individual scores for WM performance were calculated. The signal extracted from the ROIs was also used for illustration purposes (i.e., in the creation of figures) and to check whether some of the effects identified by the voxel-based correlations were driven by outliers.

\section{Results}

\section{Behavioral results}

Working memory: increased impact of emotional distraction in women, in the context of overall similar performance Analyses of the WM data showed that angry-face distractors had greater impact in women than in men in the trials associated with the highest level of confidence (LOC 3), but not in the overall WM performance (Fig. 2). The statistical analyses showed that women had lower performance to the LOC 3 trials, although no sex-related differences emerged in the overall WM performance $[t(30)=1.46, p>.1]$. LOC 3 trials had the highest proportion (60\%). A mixed-design ANOVA $($ Sex $\times$ Distractor Type $\times$ LOC) on corrected recognition scores yielded a significant main effect of $\operatorname{LOC}[F(2,56)=$ $44.38, p<.001]$, significant $\operatorname{Sex} \times \operatorname{LOC}[F(2,56)=4.1, p<$ $.02]$ and Distractor Type $\times \operatorname{LOC}[F(4,112)=6.76, p<.001]$ interactions, and a trend toward the three-way Sex $\times$ Distractor Type $\times \operatorname{LOC}[F(4,112)=1.94, p=.108]$ interaction. Further investigation to elucidate this trend-focusing on LOC 3 performance, which was justified by our previous findings in women (Denkova et al., 2010) showed that the pattern of WM impairment to emotional distraction was only present in females. Also, planned $t$ tests showed that women had lower LOC $3 \mathrm{WM}$ performance than did men for trials associated with the angry-face distractors $[t(28)=3.14, p=.004]$, but no sex-related differences were apparent in the trials associated with neutral $[t$ $(28)=1.58, p>.1]$ or scrambled $[t(28)=1.22, p>.2]$ distractors. Note that analyses performed on the data from pilot subjects $(N=42,24$ female, 18 male $)$-who had performed a similar WM task, but without distraction-

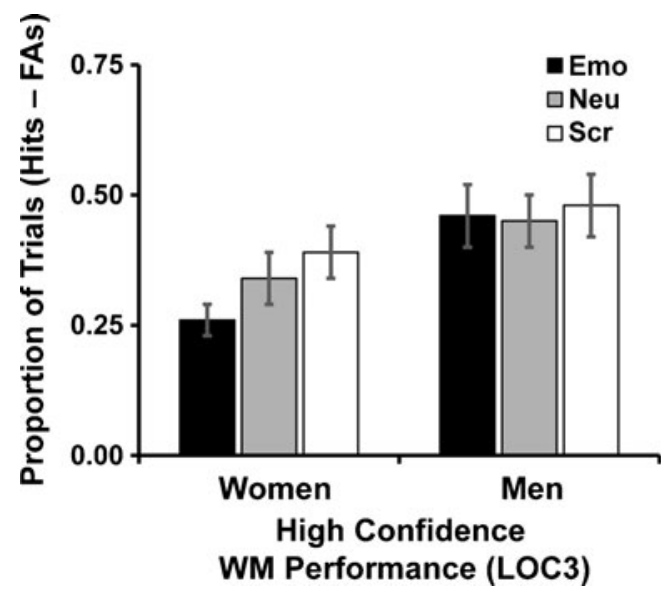

Fig. 2 Angry-face distraction impairs high-confidence responses in women. Women showed impaired WM performance for the trials associated with the highest level of confidence (LOC 3 trials; $1=$ lowest, 3 =highest), and this difference was driven by performance in the trials associated with emotional distraction. Error bars represent standard errors of the means. Emo, trials with emotional distraction; Neu, trials with neutral distraction; Scr, trials with scrambled distraction; FAs, false alarms; WM, working memory

identified no sex-related differences in using the confidence scale, for any confidence level (LOC $1, p>.3$; LOC $2, p>$ .5 ; LOC $3, p>.6$ ). This confirms that the differences between women and men in the LOC 3 ratings were specifically linked to a differential impact of the angry-face distractors on women's ability to make high-confidence ratings in the WM task, rather than to differences in the ways that they performed the LOC ratings in general.

Greater ratings of angry faces and impact on affective states in women Overall, the angry-face distractors were rated as being more emotional than the neutral-face distractors. The average emotional content scores $(1=$ lowest, $9=$ highest $)$, as rated by the subjects, were $7.02(S D=0.85)$ for the angryface distractors and $1.96(S D=0.69)$ for the neutral-face distractors. A mixed-design ANOVA (Sex $\times$ Distractor Type) on anger content ratings yielded main effects of sex $[F(1,28)=5.58, p=.025]$ and distractor type $[F(1,28)=$ $1,144.44, p<.001]$ and a significant Sex $\times$ Distractor Type interaction $[F(1,28)=7.39, p<.011]$. Post-hoc analyses showed that, as compared to men, women rated the emotional faces as being more angry $[t(28)=3.43, p<.002]$. Regarding the measures of affective states, our previous investigation in women (Denkova et al., 2010) had shown that they had significantly higher levels of state anxiety, and lower levels of state positive affect, as a result of performing the task (as identified by pre- vs. posttask differences in STAI-S and PANAS-S scores, respectively). A mixed-design ANOVA on state anxiety scores (Sex $\times$ Time) yielded a marginal effect of time $[F(1,30)=3.41, p=.075]$. Further investigation to elucidate this trend, justified by our previous findings in 
women (Denkova et al., 2010), showed that the pattern of increased state anxiety after performing the task was only present in females [women, $t(15)=2.99, p=.009$; men, $t(15)=0.29, p>$.7]. Similarly, a mixed-design ANOVA on positive affect scores (Sex $\times$ Time) yielded a main effect of Time $[F(1,30)=23.35, p<.001]$, and post-hoc tests showed that both women and men expressed a similar reduction in levels of state positive affect [women, $t(15)=2.68, p=.017$; men, $t(15)=4.18, p<.001]$. These results show that, overall, men's levels of state anxiety were less sensitive to the effect of angry-face distraction.

Together, the behavioral results suggest that female subjects were more affected by the presence of the angry-face distractors, as reflected in WM performance, emotional ratings of the distractors, and changes in posttask emotional state. Analyses of the fMRI data investigated whether these behavioral findings were linked to differences in brain activity; these analyses were performed both on all trials, as in Denkova et al. (2010), regardless of the impact of distraction on WM performance, and on trials in which sex-related differences were identified (i.e., LOC 3).

fMRI results: Sex differences in responses to emotional distraction, in the context of overall similar patterns of brain activity

To identify whether women and men show similar patterns of brain activity in response to angry-face distraction, we investigated whether the areas previously identified in women (Denkova et al., 2010) could be generalized to men. For this investigation focusing on the overall response to angryface distraction (i.e., independent of WM performance), analyses were performed on all trials. The results indicated that, indeed, the overall patterns of activity observed in men were similar to those identified in women using the same task (Denkova et al., 2010). As is illustrated in Table 1 and Fig. 3, men and women showed increased activity in response to angry-face distraction in a host of common brain regions that are involved in emotion processing (including the AMY and the vmPFC) or are susceptible to emotional modulation (e.g., FG, BA 37). By contrast, angry-face distractors evoked strong deactivations in brain regions typically involved in cognitive control and attentional processes, including dIPFC (BAs 8/9) and dorsomedial PFC (dmPFC; BA 6). These findings replicate and extend to men the results previously reported in women using similar (Dolcos et al., 2008; Dolcos et al., 2006; Dolcos \& McCarthy, 2006) or identical (Denkova et al., 2010) tasks, suggesting that men and women engage overall similar brain areas in response to negative distraction.

Increased sensitivity in the HotEmo network in women To identify brain activity specifically linked to differences in
WM performance, analyses directly comparing brain activity between women and men were performed on the trials in which sex differences were observed (i.e., LOC 3 trials). Relative to men, women showed increased activity in response to angry-face distraction in a perceptual area susceptible to emotion modulation (i.e., left FG, BA 37; see Fig. 4 and Table 2) and in a region associated with higher-level emotion processing (i.e., subgenual anterior cingulate cortex [sgACC], BA 25; Fig. 5). Brain-behavior correlations meant to further clarify these differences revealed a negative covariation between brain activity and LOC 3 WM performance in the left FG (BA 37) in women (see Table 3). These results complement our previous investigation in women (Denkova et al., 2010), in which a pattern of increased activity and negative covariation with WM performance was observed in the right FG (BA 37). Activity in the same right FG area, however, was not different and did not covary with WM performance in men. It should be noted that the findings regarding the left FG were obtained from slightly different analyses performed in the context of investigating sex differences, as compared to the initial investigation focusing on differential behavioral effects of emotional distraction within the female group (Denkova et al., 2010). Specifically, given that within the latter group emotional distractors had a greater impact on WM than did both neutral and scrambled distractors, the covariation analyses in the Denkova et al. (2010) report focused on brain areas showing differential sensitivity for emotional distractors relative to both neutral and scrambled distractors. Hence, the negative covariation in the right FG was identified in an area meeting the following conjunction criterion, dictated by the behavioral results: $[($ Emo $>\mathrm{Neu}) \cap($ Emo $>$ Scr $)]$. Similarly, in the present report, the covariation analyses were also guided by behavioral differences, but in the context of the differential effects of emotional distraction in women and men. Specifically, the negative covariation with WM performance in the left FG was identified in an area showing increased activity to emotional distractors in women relative to men, corresponding to the LOC 3 trials in which sex differences in the impact of emotional distraction on WM were identified. Thus, to ensure conceptual consistency across analyses regarding the behavioral effects, a different conjunction mask was used, which met the following criterion: $[(\mathrm{EmoF}>\mathrm{EmoM}) \cap(\mathrm{EmoF}>$ BaselineF $)]$. This was also justified by the absence of a differential impact of emotional distraction on WM in men; hence, it was not suitable to employ in men the same masking procedure that had been employed in women by Denkova et al. (2010), whose emotional distractors did differentially impact performance.

Finally, exploratory brain-behavior correlations targeting areas showing sensitivity to emotional distraction (increased or decreased activity as compared to baseline), in the 
Table 1 Common brain regions engaged in response to angry-face distraction by women and men

\begin{tabular}{|c|c|c|c|c|c|c|c|c|c|c|c|c|c|c|c|}
\hline \multicolumn{2}{|c|}{ Brain Regions } & \multirow{4}{*}{$\begin{array}{l}\text { BA } \\
\\
10\end{array}$} & \multicolumn{6}{|c|}{ Women } & \multicolumn{6}{|l|}{ Men } & \multirow{3}{*}{$\begin{array}{l}\text { Time } \\
\text { (TR) }\end{array}$} \\
\hline & & & \multicolumn{3}{|c|}{$\begin{array}{l}\text { Talairach } \\
\text { Coordinates } \\
(x, y, z)\end{array}$} & \multicolumn{2}{|l|}{$t$ Values } & \multirow{2}{*}{$\begin{array}{c}\text { Mask } \\
\begin{array}{c}\text { Emo }> \\
\text { Scr })\end{array}\end{array}$} & \multicolumn{3}{|c|}{$\begin{array}{l}\text { Talairach } \\
\text { Coordinates } \\
(x, y, z)\end{array}$} & \multicolumn{2}{|l|}{$t$ Values } & \multirow{2}{*}{$\begin{array}{c}\text { Mask } \\
\begin{array}{c}\text { Emo > } \\
\text { Scr })\end{array}\end{array}$} & \\
\hline $\begin{array}{r}\text { Increased } A \\
(\text { Emo }>S\end{array}$ & $\begin{array}{l}\text { Activity } \\
\text { cr \& Emo > Neu) }\end{array}$ & & & & & $\begin{array}{l}(\text { Emo }> \\
\text { Scr })\end{array}$ & $\begin{array}{c}(\text { Emo }> \\
\text { Neu })\end{array}$ & & & & & $\begin{array}{c}(\text { Emo }> \\
\text { Scr })\end{array}$ & $\begin{array}{c}(\text { Emo }> \\
\text { Neu })\end{array}$ & & \\
\hline \multirow[t]{2}{*}{ vmPFC $^{\dagger}$} & $\begin{array}{l}\text { R Medial } \\
\text { Frontal Gyrus }\end{array}$ & & 4 & 50 & -6 & 5.67 & 2.11 & 1.81 & - & - & - & - & - & - & 9 \\
\hline & $\begin{array}{l}\text { L Medial } \\
\text { Frontal Gyrus }\end{array}$ & 10 & - & - & - & - & - & - & -8 & 46 & -9 & 4.67 & 1.80 & 1.99 & 9 \\
\hline \multirow[t]{5}{*}{ TOC } & L Fusiform Gyrus & 37 & -44 & -59 & -7 & 5.22 & 3.56 & 9.49 & -44 & -63 & -7 & 7.09 & 5.74 & 7.63 & 9 \\
\hline & $\begin{array}{l}\text { L Inferior } \\
\text { Occipital Gyrus }\end{array}$ & 19 & -40 & -74 & -3 & 7.72 & 4.02 & 8.89 & -40 & -70 & 0 & 9.38 & 3.92 & 9.42 & 9 \\
\hline & R Fusiform Gyrus & 37 & 40 & -51 & -8 & 8.49 & 6.63 & 5.91 & 40 & -55 & -7 & 12.16 & 6.58 & 6.23 & 9 \\
\hline & $\begin{array}{l}\text { R Inferior } \\
\text { Occipital Gyrus }\end{array}$ & 19 & 40 & -70 & -3 & 8.62 & 4.71 & 9.08 & 40 & -82 & -3 & 11.77 & 3.57 & 8.62 & 9 \\
\hline & $\begin{array}{l}\text { R Middle } \\
\text { Occipital Gyrus }\end{array}$ & 19 & 48 & -77 & 11 & 6.83 & 6.21 & 7.80 & 44 & -73 & 7 & 7.94 & 5.99 & 7.55 & 9 \\
\hline Amygdala & R Amygdala & & 20 & -8 & -13 & 4.13 & 2.95 & 7.93 & 20 & -8 & -13 & 7.67 & 3.43 & 7.93 & 9 \\
\hline \multicolumn{3}{|c|}{$\begin{array}{l}\text { Decreased Activity } \\
(\text { Scr }>\text { Emo \& Neu }>\text { Emo })\end{array}$} & & & & $\begin{array}{l}(\mathrm{Scr}> \\
\text { Emo })\end{array}$ & $\begin{array}{r}\text { (Neu }> \\
\text { Emo })\end{array}$ & $\begin{array}{l}(\mathrm{Scr}> \\
\text { Emo })\end{array}$ & & & & $\begin{array}{c}(\mathrm{Scr}> \\
\text { Emo })\end{array}$ & $\begin{array}{r}(\mathrm{Neu}> \\
\text { Emo })\end{array}$ & $\begin{array}{l}(\mathrm{Scr}> \\
\text { Emo })\end{array}$ & \\
\hline \multirow[t]{2}{*}{ dlPFC } & $\begin{array}{l}\text { R Middle } \\
\text { Frontal Gyrus }\end{array}$ & 9 & 40 & 32 & 28 & 5.22 & 3.55 & 7.34 & 36 & 37 & 35 & 3.97 & 2.28 & 2.40 & 9 \\
\hline & $\begin{array}{l}\text { L Middle } \\
\text { Frontal Gyrus }\end{array}$ & 8 & -40 & 25 & 43 & 6.62 & 2.39 & 1.59 & -32 & 33 & 43 & 5.82 & 3.14 & 2.52 & 9 \\
\hline $\mathrm{dmPFC}$ & $\begin{array}{l}\text { L Medial } \\
\text { Frontal Gyrus }\end{array}$ & 6 & -4 & -9 & 59 & 7.52 & 4.08 & 2.57 & 0 & -9 & 52 & 4.21 & 2.11 & 2.28 & 9 \\
\hline LFC & $\begin{array}{l}\text { L Precentral } \\
\text { Gyrus }\end{array}$ & 6 & -59 & -3 & 11 & 5.33 & 2.87 & 2.43 & -36 & -20 & 64 & 3.48 & 2.35 & 2.40 & 8 \\
\hline \multirow[t]{2}{*}{ LTC } & $\begin{array}{l}\text { R Superior } \\
\text { Temporal Gyrus }\end{array}$ & 42 & 59 & -26 & 16 & 4.84 & 4.23 & 3.27 & 59 & -26 & 16 & 3.71 & 2.86 & 3.27 & 9 \\
\hline & $\begin{array}{l}\text { L Superior } \\
\text { Temporal Gyrus }\end{array}$ & 22 & -51 & -15 & 8 & 9.35 & 3.75 & 4.35 & -63 & -15 & 4 & 4.41 & 2.21 & 2.04 & 8 \\
\hline \multirow[t]{3}{*}{$\mathrm{mPOC}$} & $\begin{array}{l}\text { L Precuneus/ Superior } \\
\text { Parietal Lobule }\end{array}$ & 7 & -4 & -71 & 48 & 5.70 & 2.41 & 4.40 & -8 & -63 & 55 & 4.59 & 4.33 & 2.12 & 9 \\
\hline & R Cuneus & 7 & 12 & -68 & 33 & 5.89 & 3.39 & 2.92 & 8 & -72 & 33 & 4.79 & 2.58 & 3.29 & 9 \\
\hline & L Cuneus $^{\dagger \dagger}$ & 18 & 0 & -73 & 15 & 3.89 & 1.87 & 3.34 & -12 & -76 & 41 & 4.24 & 2.32 & 2.06 & 9 \\
\hline
\end{tabular}

The displayed $t$ values correspond to the peak voxels from the most dissimilar contrasts, as identified in brain areas showing activations and deactivations (i.e., Emo $>$ Scr and Scr $>$ Emo, respectively). A joint threshold of $p<.0005$ was used for each of the contributing maps, calculated independently in women and men. The results were masked by regions showing general sensitivity to negative distraction [i.e., increased (Emo $>$ $\mathrm{Scr}$ ) or decreased (Scr $>$ Emo) activity], on the basis of findings from a previous investigation (Dolcos \& McCarthy, 2006; see the Method section for details). BA, Brodmann area; TR, repetition time; Emo, emotional distractor; Scr, scrambled distractor; Neu, neutral distractor; vmPFC, ventromedial prefrontal cortex; TOC, temporo-occipital cortex; dlPFC, dorso-lateral prefrontal cortex; dmPFC, dorso-medial prefrontal cortex; LFC, lateral frontal cortex; LTC, lateral temporal cortex; mPOC, medial parieto-occipital cortex. ${ }^{\dagger}$ Although both women and men showed increased activity in adjacent vmPFC, the areas did not overlap. ${ }^{\dagger \dagger}$ Left cuneus showed relative activation to negative distraction in the mask (Emo $>$ Scr)

absence of differences between women and men, identified negative covariations with WM performance in women in areas linked to both basic emotion processing (AMY) and higher-level emotion integration and control (right ventrolateral PFC [vlPFC], BA 45/47; see Table 3). The negative covariations with LOC $3 \mathrm{WM}$ performance were identified only in female subjects and are likely indicative of impairing effects of angry-face distraction on WM performance.

Increased sensitivity in the ColdEx network and increased deactivation of the default-mode network in men Unlike the patterns observed in the ventral emotional system in women, activity in the dorsal systems of men showed patterns of decreased activity in response to angry-face distraction; similar effects were also observed in areas of the defaultmode network. Relative to women, men showed greater deactivations in response to emotional distraction in areas associated with goal-oriented processing and cognitive control, such as polar and lateral PFC (BAs 10, 9, 8, 6; see Table 2) and dorsal ACC (dACC, BA 32; see Table 2). Brain-behavior correlations meant to further clarify these differences revealed positive covariations between brain 


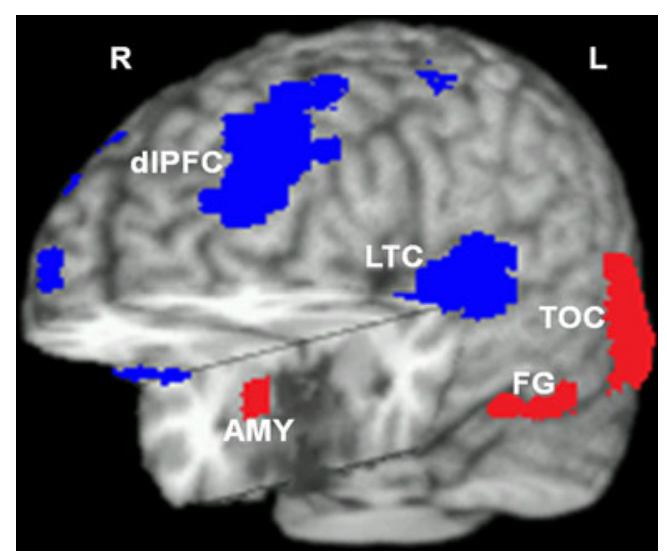

Fig. 3 Brain regions sensitive to angry-face distraction in both women and men. Common responses to emotional distraction in both women and men were identified in brain regions associated with the ventral HotEmo system (amygdala [AMY], fusiform gyrus [FG], and temporooccipital cortex [TOC]), showing increased activity to emotional distraction (Emo $>\mathrm{Neu} \cap$ Emo $>\mathrm{Scr}$ ), and in brain regions associated with the dorsal ColdEx system (dorsolateral prefrontal cortex [dIPFC] and lateral temporal cortex [LTC]), showing decreased activity to emotional distraction (Emo $<$ Neu $\cap$ Emo $<$ Scr). The cut-out of the brain reveals similar responses in women and men in the right AMY. The conjunction activation maps contributing to the overlapping activations were identified separately in male and female subjects and displayed at a joint threshold of $p<.0005$, superimposed on a highresolution brain image displayed in a tridimensional view using MRIcro (www.mccauslandcenter.sc.edu/mricro/mricro/). R , Right; L, Left

activity and LOC $3 \mathrm{WM}$ performance in the presence of emotional distraction in the right dorsal PFC (BA 6/8; Fig. 6b) and in the dorsal ACC (BA 24; see Table 3). These positive covariations with LOC $3 \mathrm{WM}$ performance were identified only in male subjects, and are likely indicative of successful engagement of mechanisms for coping with distraction.

Similar effects were also observed in a host of medial (including posterior cingulate cortex, precuneus, and cuneus; BAs 7, 31, 19), lateral parietal (BA 40), and superior temporal (BA 42) areas that partially overlap with regions of the default-mode network (Buckner et al., 2008; Raichle et al., 2001; see Table 2). Also, brain-behavior correlation analyses revealed that some of these areas showed positive covariation between activity in these regions and LOC $3 \mathrm{WM}$ performance in men (see Table 3).

Sex-related dorso-ventral hemispheric dissociation in coping with angry-face distraction in PFC Women and men showed differential patterns of activity in response to angryface distraction in ventral and dorsal lateral PFC regions, indicative of sex-related differences in engaging PFC mechanisms to cope with emotional distraction. On the one hand, although overall, women showed decreased activity to emotional distraction in a left ventral PFC region (BA 47), activity in this region was also positively correlated with WM performance in the presence of emotional distraction (Denkova et al., 2010) only in women (Fig. 6a). Thus, the same PFC region showing greater response in the female subjects who performed better in the LOC 3 showed "by default" an increased level of activity in males, who overall also had higher levels of performance in these trials. On the other hand, similar patterns of activation and covariation were identified in the right dorsal PFC (BA 6/8) in men,

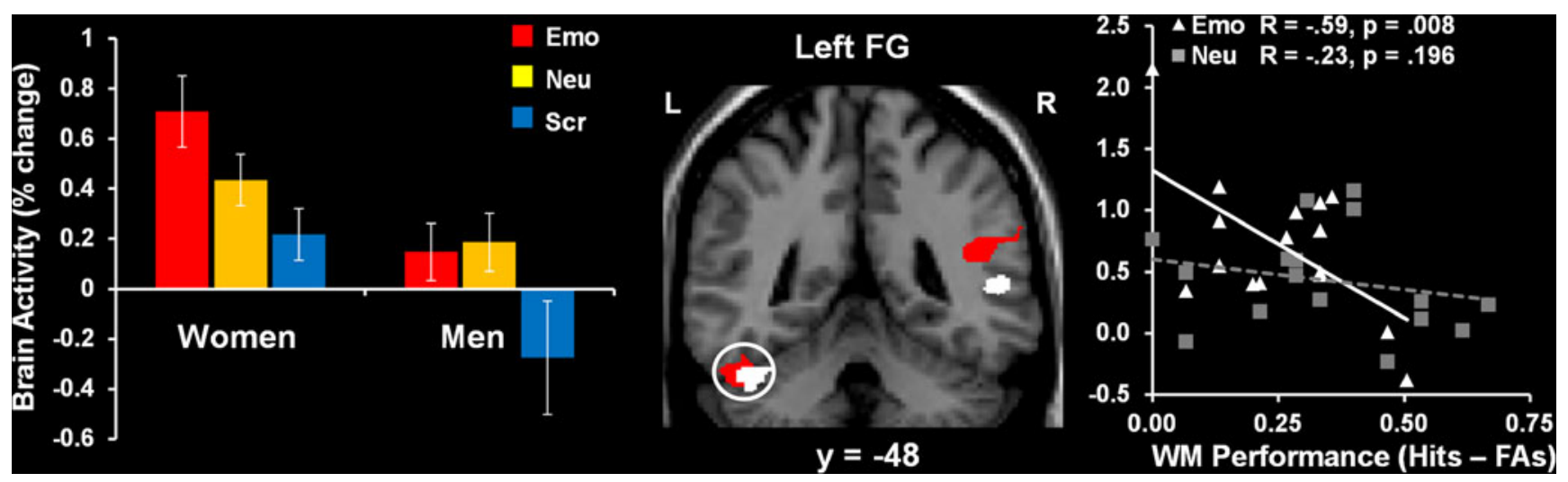

Fig. 4 Increased sensitivity to emotional distraction in left fusiform gyrus (FG) in women. Women showed increased activity in response to angry faces and negative covariation with WM performance in the left FG (BA 37). In the middle panel, the white circle on the left side indicates the FG region showing the overlap between these two effects. Inside the circle, a darker (red) area illustrates the difference in activation in response to angry-face LOC 3 trials in women versus men, masked with the main effect of emotion relative to baseline in women. The overlapping white region inside the circle illustrates the negative covariation between brain activity and WM scores for angry-face LOC 3 trials in women, masked with the main effect of emotion relative to baseline in women.
The bar graph illustrates the fMRI signal, as extracted from the region of interest (ROI) corresponding to the overlap between the two maps. The scatterplot illustrates the covariation between brain activity and LOC 3 WM scores in women, for the trials corresponding to the emotional (Emo) and neutral (Neu) distractors, as extracted from the same functional ROI. The activation maps are superimposed on a high-resolution brain image displayed in coronal view (with $y$ indicating the Talairach coordinate on the anterior-posterior axis of the brain); the joint threshold of these conjunction maps is $p<.0005$. Error bars represent standard errors of means. Emo, emotional distractors; Neu, neutral distractors; Scr, scrambled distractors; L, left; R, right 
Table 2 Sex differences in activity linked to differences in working memory performance for level-of-confidence 3 trials

\begin{tabular}{llll}
\hline Brain Regions & BA $\begin{array}{l}\text { Talairach } \\
\text { Coordinates } \\
(x, y, z)\end{array}$ & $t$ Values & Mask \\
& \\
\hline
\end{tabular}

$($ EmoF $>$ EmoM) \& $($ EmoF $>$ Baseline $)$

dmPFC Superior Frontal Gyrus

sgACC L Anterior Cingulate

LTC R Superior Temporal Gyrus

TOC L Fusiform Gyrus

R Middle Temporal Gyrus

Cerebellum L Tuber

R Declive

$($ EmoF $>$ EmoM) \& $($ EmoM $<$ Baseline $)$

dIPFC L Superior Frontal Gyrus

R Superior Frontal Gyrus

R Middle Frontal Gyrus

LFC L Superior Frontal Gyrus

R Superior Frontal Gyrus

PrCG L Precentral Gyrus

PoCG L Postcentral Gyrus

dACC L Cingulate Gyrus

LPC L Postcentral Gyrus

L Postcentral Gyrus

R Inferior Parietal Lobule

L Inferior Parietal Lobule

MPC L Superior Parietal Lobule

LTC R Superior Temporal Gyrus

L Superior Temporal Gyrus

R Middle Temporal Gyrus

PCC R Cingulate Gyrus

L Cingulate Gyrus

POC R Precuneus

mPOC

R Precuneus

L Superior Parietal Lobule/ Precuneus

L Cuneus

Cerebellum R Dentate

$($ EmoM $>$ EmoF $) \&($ EmoM $>$ Baseline $)$

vlPFC $^{\dagger} \quad$ L Middle Frontal Gyrus

TOC R Fusiform/Lingual Gyrus

MOC R Cuneus

$\begin{array}{lllllllll} & & & & (\text { EmoF }>\text { EmoM }) & (\text { EmoF }>\text { Baseline }) & (\text { Emo }>\text { Scr }) & \\ 6 & 0 & 11 & 66 & 3.32 & 4.69 & 1.95 & 9 \\ 24 / 25 & -4 & 23 & -5 & 2.73 & 3.08 & 5.16 & 9 \\ 13 & 44 & -45 & 24 & 2.59 & 4.47 & 2.64 & 9 \\ 37 & -51 & -48 & -18 & 4.22 & 4.05 & 9.62 & 9 \\ 37 & 55 & -62 & 7 & 3.19 & 4.80 & 7.79 & 9 \\ - & -51 & -48 & -21 & 3.76 & 4.04 & 5.27 & 9 \\ - & 48 & -52 & -21 & 2.22 & 4.12 & 5.48 & 9\end{array}$

$($ EmoF $>$ EmoM $) \quad($ EmoM $<$ Baseline $) \quad($ Scr $>$ Emo $)$

$\begin{array}{llllllll}10 & -24 & 47 & 5 & 3.10 & 5.15 & 5.00 & 9\end{array}$

$\begin{array}{llllllll}9 & 32 & 48 & 27 & 2.92 & 2.76 & 1.78 & 9\end{array}$

$\begin{array}{llllllll}10 & 32 & 43 & 5 & 4.06 & 5.62 & 3.27 & 9\end{array}$

$\begin{array}{llllllll}8 & -28 & 41 & 38 & 3.41 & 3.35 & 2.14 & 9\end{array}$

$\begin{array}{llllllll}6 / 8 & 36 & 10 & 47 & 3.31 & 4.21 & 2.83 & 9\end{array}$

$\begin{array}{llllllll}4 / 6 & -32 & -17 & 49 & 2.43 & 3.16 & 2.39 & 9\end{array}$

$\begin{array}{lllllll}3 & -40 & -21 & 49 & 3.48 & 4.50 & 1.96\end{array}$

$\begin{array}{llllllll}32 & -8 & 25 & 25 & 2.70 & 3.72 & 2.25 & 9\end{array}$

$\begin{array}{llllllll}40 & -51 & -23 & 16 & 2.99 & 5.84 & 5.08 & 9\end{array}$

$\begin{array}{llllllll}7 & -20 & -47 & 65 & 4.49 & 6.07 & 1.78 & 9\end{array}$

$\begin{array}{llllllll}40 & 44 & -48 & 43 & 4.90 & 5.11 & 6.46 & 9\end{array}$

$\begin{array}{llllllll}40 & -48 & -67 & 51 & 3.70 & 4.80 & 4.56 & 9\end{array}$

$\begin{array}{llllllll}7 & -44 & -71 & 51 & 3.33 & 4.99 & 3.77 & 9\end{array}$

$\begin{array}{llllllll}39 & -48 & -68 & 48 & 3.36 & 4.27 & 4.24 & 9\end{array}$

$\begin{array}{llllllll}7 & -4 & -67 & 55 & 4.16 & 5.98 & 1.88 & 9\end{array}$

$\begin{array}{llllllll}22 & 55 & -15 & 4 & 3.35 & 3.78 & 7.10 & 9\end{array}$

$\begin{array}{llllllll}22 & -51 & -15 & 4 & 3.90 & 5.19 & 4.88 & 9\end{array}$

$\begin{array}{llllllll}13 & -48 & -19 & 8 & 3.19 & 6.62 & 4.42 & 9\end{array}$

$\begin{array}{llllllll}42 & -55 & -23 & 16 & 2.98 & 6.77 & 3.73 & 9\end{array}$

$\begin{array}{llllllll}21 & 67 & -35 & -2 & 4.08 & 4.33 & 1.26 & 9\end{array}$

$\begin{array}{llllllll}31 & 12 & -21 & 38 & 3.59 & 3.34 & 2.04 & 9\end{array}$

$\begin{array}{llllllll}31 & -4 & -44 & 43 & 3.59 & 3.89 & 2.98 & 9\end{array}$

$\begin{array}{llllllll}19 & 40 & -72 & 40 & 4.49 & 6.31 & 5.14 & 9\end{array}$

$\begin{array}{llllllll}7 & 4 & -63 & 51 & 2.71 & 3.52 & 2.27 & 9\end{array}$

$\begin{array}{llllllll}7 & -4 & -67 & 55 & 4.16 & 5.98 & 1.88 & 9\end{array}$

$\begin{array}{llllllll}19 & -8 & -80 & 30 & 2.54 & 4.57 & 2.02 & 9\end{array}$

$\begin{array}{lllllll}- & 20 & -56 & -24 & 3.07 & 3.35 & 2.51\end{array}$

$($ EmoM $>$ EmoF $) \quad($ EmoM $>$ Baseline $) \quad($ Emo $>$ Scr $)$

$\begin{array}{llllllll}47 & -48 & 42 & -5 & 3.39 & 2.71 & 3.38 & 9 \\ 19 & 32 & -74 & -10 & 2.90 & 5.23 & 9.36 & 9 \\ 19 & 16 & -96 & 27 & 2.93 & 5.79 & 3.80 & 9\end{array}$

The $t$ values displayed correspond to the peak voxels from the between-groups comparisons (i.e., women vs. men). A joint threshold of $p<.0005$ was used for the conjunction maps identifying differences between women and men. The results were masked by regions showing general sensitivity to negative distraction [i.e., increased (Emo $>$ Scr) or decreased (Scr $>$ Emo) activity], on the basis of findings from a previous investigation (Dolcos \& McCarthy, 2006; see the Method section for details). BA, Brodmann area; TR, repetition time; Emo, emotional distractor; Scr, scrambled distractor; Neu, neutral distractor; dmPFC, dorso-medial prefrontal cortex; sgACC, subgenual anterior cingulate cortex; LTC, lateral temporal cortex; TOC, temporo-occipital cortex; dIPFC, dorso-lateral PFC; LFC, lateral frontal cortex; PrCG, precentral gyrus; PoCG, postcentral gyrus; dACC, dorsal ACC; LPC, lateral parietal cortex; MPC, medial parietal cortex; PCC, posterior cingulate cortex; POC, parieto-occipital cortex; mPOC, medial POC; vlPFC, ventro-lateral PFC; MOC, medial occipital cortex. ${ }^{\dagger}$ Activity in the left ventro-lateral PFC (five voxels), a typical HotEmo area, was identified using the (Scr > Emo) mask (Dolcos \& McCarthy, 2006), due to a specific pattern of deactivation in this region in women 


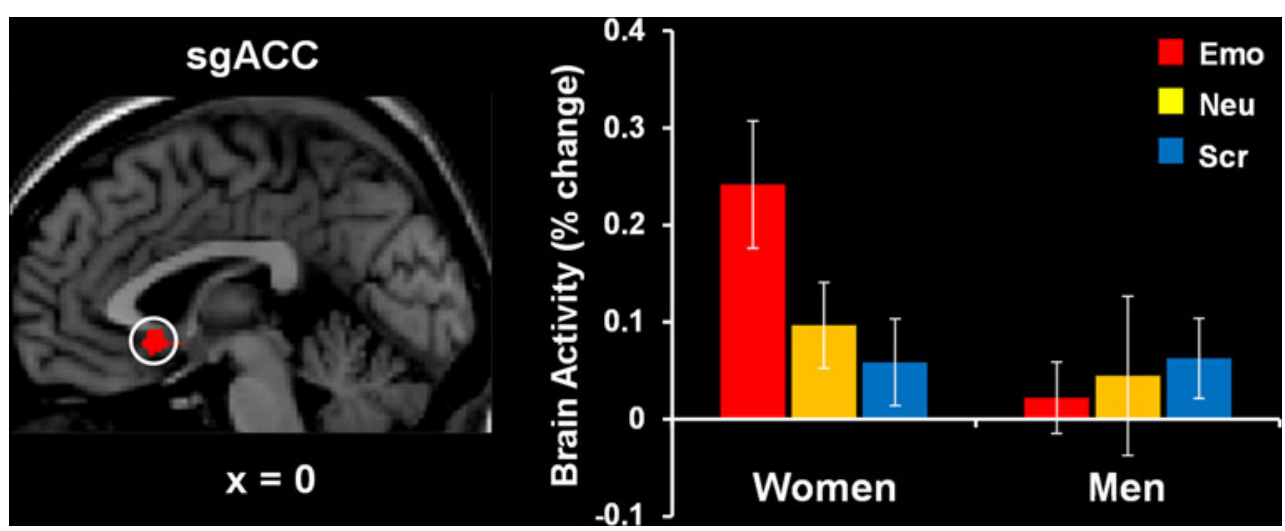

Fig. 5 Increased activity in the subgenual anterior cingulate cortex ( $\operatorname{sgACC})$ in women. Women showed an increased response to emotional distraction in the sgACC (BA 25). The area indicated by the white circle illustrates the difference in activation in response to angry faces in women versus men, masked with the main effect of emotion relative to baseline in women. The bar graph illustrates the fMRI signal, as extracted from the region of interest corresponding to the

where-although overall they showed decreased activity to emotional distraction - activity was also positively correlated with WM performance in the presence of emotional distraction only in men (Fig. 6b).

\section{Discussion}

The goals of the present study were to determine whether sex differences in basic emotional reactivity are associated with differences in emotional distractibility and to identify the neural mechanisms that implement differences in emotional distractibility between women and men. We identified three main findings. First, our behavioral results showed an increased impact of emotional distraction in women. This was reflected in increased impact of angry-face distractors for WM trials associated with a high level of confidence, increased emotional ratings of the distractors, and increased change in negative affect in the posttask emotional states. Second, brain-imaging results identified dissociable patterns of activity in the HotEmo and ColdEx networks in women and men, in the context of similar overall patterns of response to emotional distraction in the two sexes. Specifically, women showed increased sensitivity to emotional distraction in regions of the HotEmo system, including both emotion-processing regions (AMY, vlPFC, and sgACC) and regions susceptible to affective modulation (FG), whereas men showed increased sensitivity in regions of the ColdEx system, including polar and dorsal PFC and dorsal ACC, and in brain regions associated with the default-mode network. Finally, we also found a dorsalventral hemispheric dissociation within the lateral PFC linked to the engagement of mechanisms to cope with emotional distraction, with left ventral PFC activity being difference in activation between women and men. The activation map is superimposed on a high-resolution brain image displayed in sagittal view (with $x$ indicating the Talairach coordinate on the left-right axis of the brain); the joint threshold of the conjunction maps is $p<.0005$. Error bars represent standard errors of means. Emo, emotional distractors; Neu, neutral distractors; Scr, scrambled distractors

linked to individual differences in WM performance in women, and right dorsal PFC activity being linked to individual differences in WM performance in men. These findings are discussed in turn below.

Behavioral results: Increased impact of emotional distraction in women, in the context of overall similar WM performance

The present findings showing enhanced overall sensitivity in women in response to emotional distraction are consistent with evidence of increased reactivity among women to emotional stimuli, in general (e.g., Kring \& Gordon, 1998), and to emotional facial expressions, in particular (Dimberg \& Lundquist, 1990; Orozco \& Ehlers, 1998). It should be noted, however, that no differences were observed in the overall WM performance between men and women, but rather in the responses associated with the highest level of confidence. This suggests that sex-related differences in the response to emotional challenge, as induced by our task with distraction, are more subtle and affect only some aspects of behavior - that is, the specific impact on responses requiring a higher level of confidence in the WM task (LOC 3). Although this raises the possibility of sexrelated differences in the ways that the LOC ratings were performed by women and men in general, this does not seem to be the case, as shown by analyses comparing LOC responses in women and men who performed a similar task, but without distraction. These findings confirm that the differences between women and men in the LOC 3 ratings were specifically linked to a differential impact of the angry-face distractors on their ability to make high-confidence ratings in the WM task, and they validate the LOC ratings as being more sensitive than are 
Table 3 Covariation between brain activity and level-of-confidence 3 working memory performance in women and men

\begin{tabular}{|c|c|c|c|c|c|c|c|c|c|c|c|}
\hline \multirow{2}{*}{\multicolumn{2}{|c|}{ Brain Regions }} & \multirow[t]{2}{*}{$\mathrm{BA}$} & \multicolumn{3}{|c|}{$\begin{array}{l}\text { Talairach Coordinates } \\
(x, y, z)\end{array}$} & \multicolumn{3}{|c|}{$\begin{array}{l}\text { Correlations } \\
(r \text { Values })\end{array}$} & \multirow[t]{2}{*}{$t$ Values } & \multirow[t]{2}{*}{ Mask } & \multirow[t]{2}{*}{$\begin{array}{l}\text { Time } \\
\text { (TR) }\end{array}$} \\
\hline & & & & & & Emo & $\mathrm{Neu}$ & Scr & & & \\
\hline \multicolumn{6}{|c|}{$\begin{array}{l}\text { Women: Negative covariation \& } \\
\text { (EmoF > Baseline) }\end{array}$} & & & & $($ EmoF $>$ Baseline $)$ & $($ Emo $>$ Scr $)$ & \\
\hline vlPFC & R Inferior Frontal Gyrus & $45 / 47$ & 55 & 24 & 6 & $-.73^{* * *}$ & -.31 & -.21 & 4.37 & 7.42 & 9 \\
\hline \multirow[t]{2}{*}{ LTC } & R Superior Temporal Gyrus & 38 & 32 & 3 & -17 & $-.65^{* * *}$ & -.39 & .13 & 3.17 & 3.53 & 9 \\
\hline & & 22 & 51 & -46 & 10 & $-.59^{* *}$ & -.23 & -.02 & 3.92 & 3.71 & 9 \\
\hline \multirow[t]{4}{*}{ TOC } & R Fusiform Gyrus & $19 / 37$ & 32 & -66 & -7 & $-.68^{* * *}$ & .03 & -.26 & 3.71 & 9.27 & 9 \\
\hline & L Fusiform Gyrus & 37 & -48 & -48 & -18 & $-.58^{* *}$ & -.34 & $-.49^{*}$ & 5.40 & 10.06 & 9 \\
\hline & R Middle Temporal Gyrus & 37 & 48 & -62 & 3 & $-.67^{* * *}$ & .07 & -.36 & 3.99 & 5.89 & 9 \\
\hline & R Inferior Temporal Gyrus & 37 & 48 & -66 & -3 & $-.69^{* * *}$ & -.07 & -.23 & 4.23 & 7.25 & 9 \\
\hline \multirow[t]{3}{*}{ LatOC } & R Middle Occipital Gyrus & 18 & 28 & -97 & 12 & $-.52^{*}$ & .21 & .13 & 6.54 & 5.47 & 9 \\
\hline & L Middle Occipital Gyrus & $19 / 18$ & -40 & -92 & 16 & $-.61^{* *}$ & -.28 & -.33 & 5.01 & 9.41 & 9 \\
\hline & L Inferior Occipital Gyrus & 18 & -24 & -90 & -6 & $-.68^{* * *}$ & -.23 & -.2 & 3.15 & 5.36 & 9 \\
\hline \multirow[t]{2}{*}{$\mathrm{MOC}$} & R Lingual Gyrus & 17 & 8 & -89 & 4 & $-.76^{* * *}$ & .09 & .41 & 4.27 & 5.05 & 9 \\
\hline & R Cuneus & $17 / 18$ & 8 & -93 & 5 & $-.86^{* * *}$ & -.003 & -.29 & 5.19 & 5.98 & 9 \\
\hline \multirow[t]{2}{*}{ MTL } & R Amygdala/Uncus & & 28 & -1 & -17 & $-.62^{* *}$ & -.11 & .03 & 3.96 & 5.79 & 9 \\
\hline & L Parahippocampal Gyrus & 27 & -20 & -31 & -2 & $-.66^{* * *}$ & -.12 & -.23 & 3.34 & 6.17 & 9 \\
\hline \multirow{2}{*}{\multicolumn{2}{|c|}{$\begin{array}{l}\text { Cerebellum R Declive } \\
\text { Men: Positive covariation } \& \\
\text { (EmoM }<\text { Baseline) }\end{array}$}} & - & 32 & -63 & -7 & $-.78^{* * *}$ & -.23 & -.33 & 3.21 & 7.82 & 9 \\
\hline & & & & & & & & & (EmoM $<$ Baseline $)$ & $(\mathrm{Scr}>\mathrm{Emo})$ & \\
\hline \multirow[t]{2}{*}{ LFC } & R Superior Frontal Gyrus & 6 & 16 & -8 & 67 & $.77^{* * *}$ & .32 & .38 & 5.15 & 2.12 & 9 \\
\hline & R Middle Frontal Gyrus & $6 / 8$ & 28 & 18 & 54 & $.64^{* *}$ & .31 & -.03 & 4.72 & 3.24 & 9 \\
\hline MFC & R Medial Frontal Gyrus & 6 & 12 & -17 & 49 & $.78^{* * *}$ & -.18 & .02 & 3.41 & 3.89 & 9 \\
\hline PcL & L Paracentral Lobule & 31 & -4 & -25 & 45 & $.69^{* * *}$ & .17 & -.21 & 4.99 & 2.28 & 9 \\
\hline \multirow[t]{2}{*}{$\mathrm{dACC}$} & L Cingulate Gyrus & 24 & -4 & -6 & 44 & $.52^{*}$ & .17 & .15 & 3.70 & 1.93 & 9 \\
\hline & R Cingulate Gyrus & 24 & 4 & -6 & 44 & $.56^{*}$ & .12 & .27 & 3.17 & 1.84 & 9 \\
\hline \multirow[t]{2}{*}{ LTC } & L Superior Temporal Gyrus & 22 & -51 & -15 & 4 & $.56^{*}$ & .23 & .05 & 5.19 & 4.88 & 9 \\
\hline & L Transverse Temporal Gyrus & 42 & -55 & -15 & 12 & $.83^{* * *}$ & .36 & .27 & 5.62 & 2.51 & 9 \\
\hline \multirow[t]{2}{*}{$\mathrm{mPOC}$} & L Precuneus & 7 & -4 & -68 & 44 & $.76^{* * *}$ & .37 & -.01 & 4.92 & 5.18 & 9 \\
\hline & R Precuneus & 7 & 4 & -64 & 44 & $.65^{* *}$ & .21 & -.22 & 3.18 & 2.28 & 9 \\
\hline Cerebellum & R Declive & - & 8 & -59 & -14 & $.76^{* * *}$ & .28 & .05 & 4.31 & 2.64 & 9 \\
\hline \multicolumn{2}{|c|}{$\begin{array}{l}\text { Women: Positive covariation \& } \\
\text { (EmoF }<\text { Baseline) }\end{array}$} & & & & & & & & $($ EmoF $<$ Baseline $)$ & $(\mathrm{Scr}>\mathrm{Emo})$ & \\
\hline LTC & L Inferior Temporal Gyrus & 20 & -63 & -28 & -19 & $.61^{* *}$ & -.08 & $.62^{* *}$ & 3.20 & 4.15 & 9 \\
\hline
\end{tabular}

The displayed $r$ and $t$ values correspond to the peak voxels identified by brain-behavior correlations for the emotional level-of-confidence 3 trials. A joint threshold of $p<.0005$ was used for each of the conjunction maps. The results were masked by regions showing general sensitivity to negative distraction [i.e., increased (Emo $>$ Scr) or decreased (Scr $>$ Emo) activity], on the basis of findings from a previous investigation (Dolcos \& McCarthy, 2006; see the Method section for details). BA, Brodmann area; TR, repetition time; Emo, emotional distractor; Scr, scrambled distractor; Neu, neutral distractor; vlPFC, ventro-lateral PFC; LTC, lateral temporal cortex; TOC, temporo-occipital cortex; LatOC, lateral occipital cortex; MOC, medial OC; MTL, medial temporal lobe; LFC, lateral frontal cortex; MFC, medial frontal cortex; PcL, paracentral lobule; dACC, dorsal anterior cingulate cortex; mPOC, medial parieto-occipital cortex. ${ }^{*} p<.05,{ }^{* *} p<.01,{ }^{* * *} p<.005$

old/new responses in assessing subtle differences in the impact of emotional distraction.

It may seem somewhat surprising that negative emotional distractors selectively impaired high-confidence responses, given that emotional stimuli often confer a selective benefit on response accuracy (Dolcos, LaBar, \& Cabeza, 2005; Ochsner, 2000; Sharot, Delgado, \& Phelps, 2004). It should be noted, however, that this effect is typically observed when the emotional information is task-relevant and is associated with enhanced episodic memory performance. Instead, in the present study, the emotional information was task-irrelevant, so that the typical effect would be impaired performance in the main task (e.g., in WM: Anticevic, Barch, \& Repovs, 2010; Dolcos et al., 2008; Dolcos \& McCarthy, 2006). Hence, it is actually expected that this detrimental effect would also impair the ability to make correct responses with increased confidence. This is consistent with the idea presented in the introduction, that activity in the 


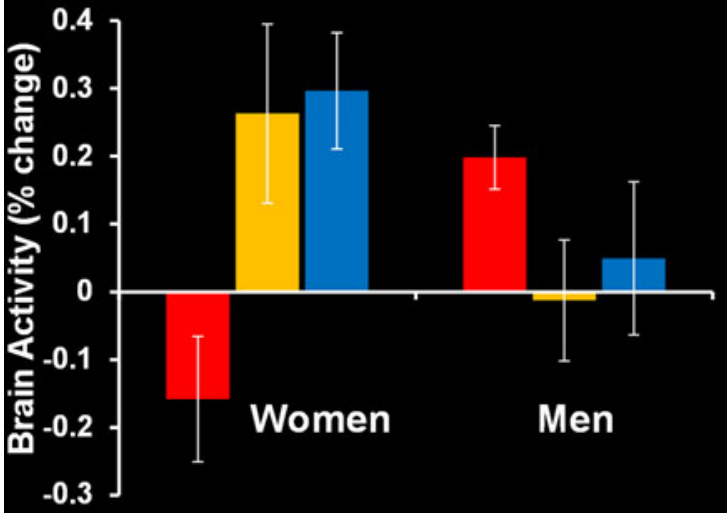

Women

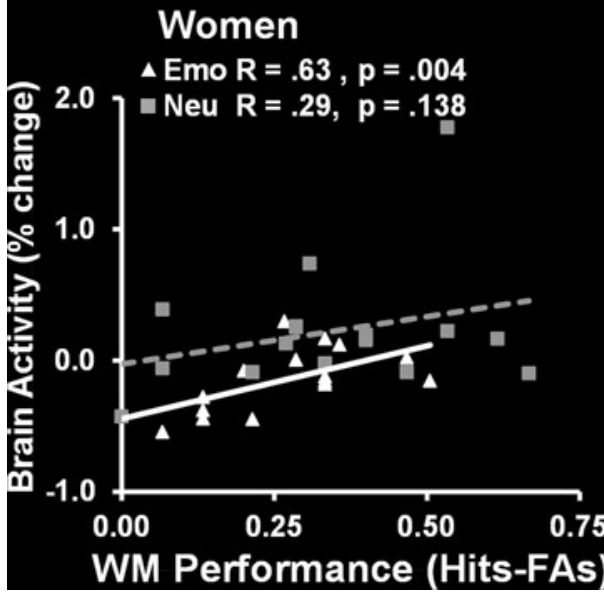

$\mathbf{L}$

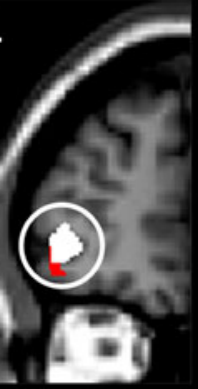

$y=43$

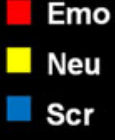

Scr

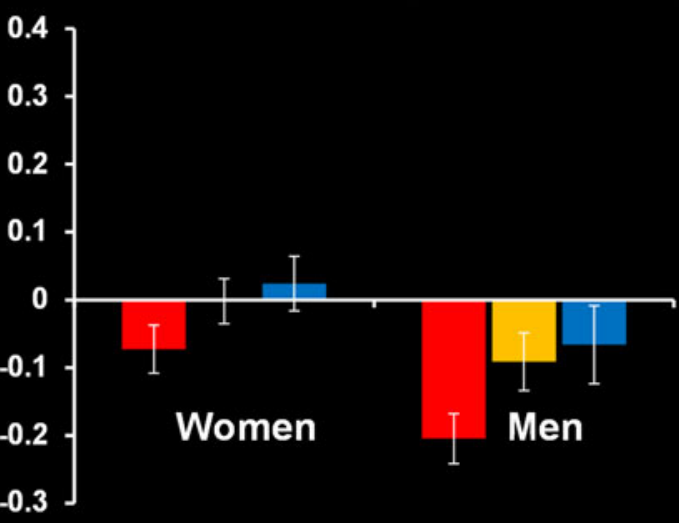

$\mathbf{R}$

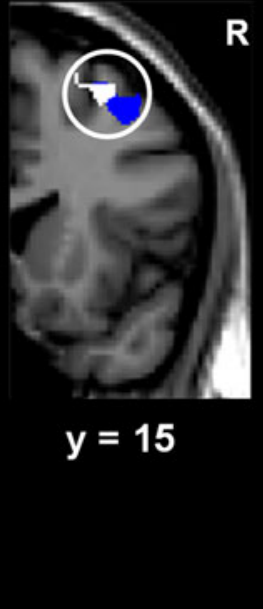

Men

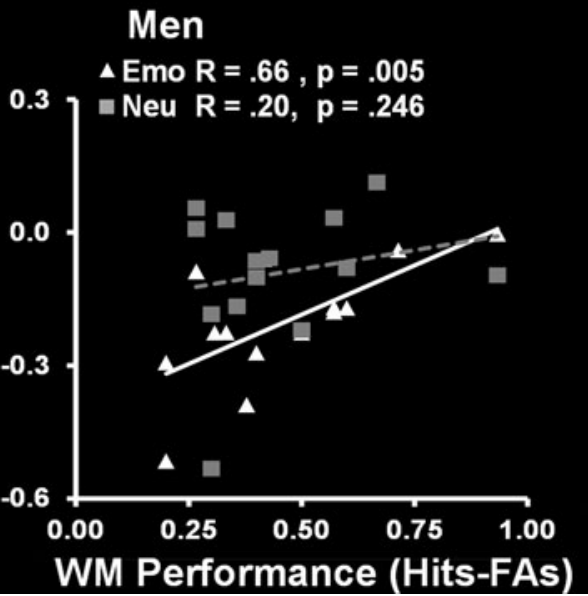

Fig. 6 Sex-related dorso-ventral dissociation in the lateral prefrontal cortex (PFC) in response to emotional distraction, linked to WM performance. The left lateral PFC (BA 47) had overall reduced activity in women but showed increased activity in those women who coped successfully with emotional distraction (A); a similar pattern was observed in the right lateral PFC (BA 8/6) in men-although they overall showed reduced activity in this region as compared to women, those who had increased activity also coped successfully with emotional distraction (B). (A) The bar graph illustrates the fMRI signal, as extracted from the region of interest (ROI) corresponding to the difference in activation between women and men. The ROI is located inside the left circle, in the central panel, and illustrated with a darker color (red). This area showed greater activation to angry faces in men than in women, masked with the effect of emotion relative to baseline activity in men. The overlapping white area inside the same circle illustrates the positive covariation between brain activity and LOC 3 WM performance in women, in an area sensitive to emotional distraction (see Fig. 5 in Denkova et al., 2010). The scatterplot illustrates the covariation between brain activity and LOC $3 \mathrm{WM}$ scores in women for the trials corresponding to the emotional (Emo) and neutral (Neu) distractors, as extracted from the ROI meeting the conjunction criteria (Denkova et al., 2010). (B) The bar graph illustrates the fMRI signal, as extracted from the ROI corresponding to the overlap between the two maps located inside the white circle on the right side, in the central panel. This circle surrounds a darker area (blue), illustrating greater deactivation to angry faces in men than in women, masked with the effect of emotion relative to baseline activity in men. The overlapping white area inside the same circle illustrates the positive covariation between brain activity and LOC $3 \mathrm{WM}$ scores in men, for the trials corresponding to the emotional distractors, masked with the effect of emotion relative to baseline. The scatterplot illustrates the covariation between brain activity and LOC 3 WM scores in men for the trials corresponding to the emotional (Emo) and neutral (Neu) distractors, as extracted from the ROI corresponding to the overlap of the two maps. The conjunction activation maps are displayed at a joint threshold of $p<.0005$, superimposed on a high-resolution brain image displayed in coronal view (with $y$ indicating the Talairach coordinates on the anterior-posterior axis of the brain). Error bars represent standard errors of means. Emo, emotional distractors; Neu, neutral distractors; Scr, scrambled distractors; L, left; R, right affective and executive neural systems is strongly interconnected, such that increased activity in the ventral affective regions disrupts activity in the dorsal system and results in cognitive impairment. The selective impairment of high-confidence responses, as opposed to the general detrimental effect observed in other studies (e.g., Dolcos \& McCarthy, 2006), could be explained by the milder emotional content conveyed by the angry faces used in the present study, as compared to the high-arousing negative pictures from the International Affective Picture System (Lang et al., 2008) used in previous studies. 
Differential sensitivity in women and men was also reflected in higher ratings of angry distractors and increased changes on posttask negative affective state in women, which further confirms that women were more affected overall by the angry faces used as distractors in the present task. Sex differences in ratings of angry faces are consistent with the idea of increased emotional sensitivity in women. However, an alternative way of looking at sex differences in neural responses would be to equate the behavioral performance in women and men, to make sure that eventual differences at the neural level would be observed in the absence of behavioral differences. Thus, one might argue that in such a context, the self-report measure is not a manipulation check, but simply another measure of gender differences that may be viewed as a confound. However, this was not the case in the present investigation, in which, on the basis of established differences between women and men in some aspects of emotion processing (i.e., basic emotional reactivity), we investigated whether these differences "translate" into differences in other related aspects (i.e., emotional distractibility), and whether such possible differences in behavior are associated with similar or different neural correlates. Hence, the higher ratings of angry faces observed in women were not a failed manipulation check, but an expected result. Overall, these behavioral findings warranted analyses of brain-imaging data examining both common patterns of activation and responses linked to the differential impacts of emotional distraction in women and men, as we will discuss below.

fMRI results: Sex differences in responses to emotional distraction, in the context of overall similar patterns of brain activity

Complementing our previous investigation (Denkova et al., 2010), analyses performed on all trials showed that men and women display similar patterns of activation and deactivation in a host of brain regions associated with the ventral HotEmo (e.g., AMY, vmPFC, and FG) and dorsal ColdEx (e.g., dlPFC) neural systems in response to emotional distractors inducing specific emotions (i.e., anxiety). These findings confirm previous results that have identified this as a robust pattern of activity in response to distractors inducing general negative affect in both healthy (Anticevic et al., 2010; Chuah et al., 2010; Denkova et al., 2010; Dolcos et al., 2008; Dolcos \& McCarthy, 2006; Dolcos et al., 2007) and clinical (Anticevic, Repovs, Corlett, \& Barch, 2011; Diaz et al., 2011; Morey et al., 2009) groups. However, unlike previous investigations, which did not investigate sex differences, in the present study we also identified differences in brain activity linked to differential impacts of emotional distraction in women and men.
Increased sensitivity in the HotEmo network in women In addition to areas identified in the right FG showing increased activation to and negative covariation with WM performance for emotional distraction, in the present investigation we also identified other brain regions showing increased similar engagement in women. These areas included bilateral FG (BA 37), as well as areas associated with basic emotional response (AMY), higher-level emotion integration (i.e., sgACC, BA 25), and areas associated with top-down control of emotion (i.e., right vlPFC, BA 47). However, the left ventral PFC (BA 47) showed a pattern of positive covariation with WM performance in women, probably reflecting the engagement of mechanisms to successfully cope with distraction, as we will also discuss in the next section.

Related to the pattern of activity in the FG, it may seem surprising at first that we observed a negative covariation that was selective for angry faces in a perceptual area (left FG), given the possibility that both emotional and neutral distractors could capture attention and lead to WM impairment. It should be noted, however, that this effect was identified in an area specifically showing sensitivity to emotional distraction (i.e., increased activity) and differences in activation between women and men. Therefore, it is more likely that the covariation with WM performance observed in these areas would be identified for the items also producing differential effects in activation (i.e., emotional LOC 3). Although this does not exclude the possibility of also identifying covariations between WM performance and activations for neutral distractors, in the FG or in other areas producing "bottom-up" effects, the absence of such strong covariations for the neutral items may reflect the fact that these distractors did not actually produce an impairment in WM performance in either women or men. Hence, this effect is consistent with the specific impairment observed in women only (Denkova et al., 2010), and with the idea of a "bottom-up" impact of emotional distraction.

Of particular note is the fact that women showed an increased specific response to emotional distraction in the sgACC (BA 25). This region of the ACC is an area closely tied to the autonomic nervous system, which has been linked to the experience of negative emotion in both healthy and clinical samples (Baeken et al., 2010; Ball et al., 2012; Gotlib et al., 2005; Mobbs et al., 2009). Sex-related differences in sgACC have been previously reported, with increased activation in women possibly linked to enhanced emotional reactivity or stronger autonomic reactions to emotional stimuli (Wager, Phan, Liberzon, \& Taylor, 2003). For example, using an instructed fear/anticipatory anxiety paradigm, Butler et al. (2005) reported increased activity in the sgACC in women relative to men during anticipation of mild electric shock. However, to our knowledge, this effect 
has not been previously studied in relation to transient angry-face distraction.

Altogether, these findings, particularly the patterns of negative covariation in the FG and AMY are consistent with a bottom-up impact of angry-face distraction, and the negative covariation in the right $\mathrm{VlPFC}$ is consistent with unsuccessful engagement of top-down control in the face of emotional distraction. These results provide support for the idea that females may be more sensitive to angry-face distraction and offer insight into possible overlapping mechanisms between transient and longer-state moods that may be involved in emotional dysfunctions associated with affective disorders (e.g., Drevets \& Raichle, 1998; Mayberg, 1997).

Increased sensitivity in the ColdEx network and increased deactivation of the default-mode network in men Consistent with the idea of increased sensitivity in the ColdEx network, men showed reduced activity in the polar (BA 10) and lateral (BAs 9, 8, 6) PFC, in response to angry-face distractors. The pattern of PFC deactivation in men suggests increased interference with maintenance of goal-relevant information in WM in the presence of emotional distraction (D'Esposito et al., 2000; Levy \& Goldman-Rakic, 2000; Miller \& Cohen, 2001). Preliminary analyses confirmed that the differential effects of the distractors were most robust 14-16 s after memoranda onset (TR 9) in the present data. Moreover, men also showed a pattern of nonspecific deactivations in the dorsal ACC (BA 32). This suggests a more general impact of distraction on top-down cognitive control mechanisms (Bush, Luu, \& Posner, 2000), although this was not reflected in differences in WM performance. However, both right lateral PFC (BA 6/8) and dorsal ACC (BA 24) also showed a pattern of positive covariation with WM performance, probably reflecting the engagement of mechanisms to successfully cope with distraction.

A similar pattern of increased deactivations was observed in areas of the posterior midline (BAs 7,31), lateral parietal (BA 40), and superior temporal (BA 42) regions, which are partially overlapping with regions of the default-mode network. The default-mode network is characterized by increased resting-state activity and by deactivation in tasks that require attention to external stimuli (Buckner et al., 2008; Raichle et al., 2001). Thus, the present findings of increased deactivation in men could indicate that men were more successful in disengaging the default-mode network as a result of the demand to perform goal-directed processing, and consequently performed better in the WM task (McKiernan, Kaufman, Kucera-Thompson, \& Binder, 2003; Raichle et al., 2001). Alternatively, given that these effects were observed for trials associated with increased WM performance in men relative to women, the specific pattern of deactivation may be indicative of sex-related differences in the strategies employed by men and women in response to distraction.

Sex-related dorsal-ventral hemispheric dissociation in coping with angry-face distraction in the PFC The present investigation also revealed a dorsal-ventral hemispheric dissociation in the lateral PFC between women and men coping with emotional distraction. Specifically, although the left ventral PFC (BA 47) showed reduced activity in women than in men, it also showed a positive covariation with WM performance in women, suggesting its engagement by women who successfully coped with emotional distraction (Denkova et al., 2010). Consistent with this idea, previous evidence has consistently linked activity in this region to coping with emotional distraction (Banich et al., 2009; Dolcos et al., 2008; Dolcos et al., 2006). By contrast, although the right dorsal PFC (BA 6/8) showed reduced activity in men relative to women, it also showed a positive covariation with WM performance in men, suggesting its engagement by men who also successfully coped with emotional distraction. Similar effects were also found in the dorsal ACC, indicating that males who maintained an increased level of activity in these areas, despite overall reduced activity as compared to females, also showed better WM performance. These results are consistent with evidence linking activity in lateral BAs 6 and 8 with WM processing, especially in the spatial domain (Courtney, Petit, Maisog, Ungerleider, \& Haxby, 1998; D’Esposito, 2008; Tanaka, Honda, \& Sadato, 2005), and activity in the dorsal ACC with engagement of top-down executive control (Bush et al., 2000).

Interestingly, analyses of brain-behavior relationships in general revealed sex-related opposing patterns of covariation in women and men (i.e., more negative brain-behavior correlations in women and more positive correlations in men). However, this apparently unexpected dissociation could be explained by the fact that these covariations were investigated in areas specifically showing sensitivity to emotional distraction, and in some cases, also showing differences in activation between women and men. The pattern of negative covariation with WM performance in women was generally observed in brain regions of the ventral affective system, which also showed increased activity to angry-face distractors in women. Hence, these negative covariations are consistent with the idea that increased sensitivity in HotEmo areas is linked to impaired WM performance in women. Note that women also showed positive covariation in the left ventral PFC (BA 47), consistent with engagement of this brain region in coping with emotional distraction (Denkova et al., 2010). On the other hand, the pattern of positive covariations in dorsal areas in men is consistent with less of an overall impact of negative distraction, as compared to women, possibly due to increased engagement of these regions 
to maintain WM performance in the presence of emotional distraction. Overall, these findings suggest that the increased impact of emotional distraction in women is linked to "bottom-up" effects in the HotEmo system, whereas increased resilience to emotional distraction in men is linked to "top-down" effects in the ColdEx system.

\section{Conclusions}

In summary, the present study has provided evidence for sex differences in the context of similar behavioral and brain responses to negative distractors in women and men. Our results suggest that enhanced emotional competence in women may have the side effect of increased emotional reactivity, which in turn may lead to enhanced emotional distractibility, although the present findings do not establish that direction for such a causal relationship. Behavioral results showed an increased impact of emotional distraction in women, which was reflected in decreased WM performance that was specific to trials associated with high-confidence responses, increased emotional ratings of distractors, and increased posttask negative affect. Our fMRI findings identified sex differences in patterns of activity in the HotEmo and ColdEx networks, in the context of overall similar responses to emotional distraction in women and men. The similarities in the engagement of the ventral-affective and dorsal-executive systems suggest that men and women deploy similar general mechanisms in response to transient emotional distraction. The sex-related dissociations are consistent with increased sensitivity in "bottom-up" responses in women, linked to impaired WM performance, and increased sensitivity in "top-down" responses in men, linked to increased performance, in the face of emotional distraction. Finally, the study also revealed a dorsal ventral hemispheric dissociation within the lateral PFC that was linked to the engagement of mechanisms to cope with emotional distraction, with the left ventral PFC being linked to individual differences in WM performance in women, and the right dorsal PFC being linked to individual differences in men. These results contribute to a better understanding of sex differences in responses to emotional distraction in healthy behavior, and have implications for understanding factors that may influence susceptibility to affective disorders.

Acknowledgments This research was supported by a Grant from the University Hospital Foundation and from start-up funds (to F.D.). The authors thank Keen Sung, Gloria Wong, Kristen Sabourin, and Kristina Suen for assistance with stimulus creation and data analysis, and DLab members for help with manuscript preparation.

\section{References}

Andrews-Hanna, J. R., Reidler, J. S., Sepulcre, J., Poulin, R., \& Buckner, R. L. (2010). Functional-anatomic fractionation of the brain's default network. Neuron, 65, 550-562.

Anticevic, A., Barch, D. M., \& Repovs, G. (2010). Resisting emotional interference: Brain regions facilitating working memory performance during negative distraction. Cognitive, Affective, \& Behavioral Neuroscience, 10, 159-173. doi:10.3758/ CABN.10.2.159

Anticevic, A., Repovs, G., Corlett, P. R., \& Barch, D. M. (2011). Negative and nonemotional interference with visual working memory in schizophrenia. Biological Psychiatry, 70, 1159-1168.

Baeken, C., Van Schuerbeek, P., De Raedt, R., Ramsey, N. F., Bossuyt, A., De Mey, J., \& Luypaert, R. (2010). Reduced left subgenual anterior cingulate cortical activity during withdrawal-related emotions in melancholic depressed female patients. Journal of Affective Disorders, 127, 326-331.

Ball, T. M., Sullivan, S., Flagan, T., Hitchcock, C. A., Simmons, A., Paulus, M. P., \& Stein, M. B. (2012). Selective effects of social anxiety, anxiety sensitivity, and negative affectivity on the neural bases of emotional face processing. NeuroImage, 59, 1879-1887.

Banich, M. T., Mackiewicz, K. L., Depue, B. E., Whitmer, A. J., Miller, G. A., \& Heller, W. (2009). Cognitive control mechanisms, emotion and memory: A neural perspective with implications for psychopathology. Neuroscience and Biobehavioral Reviews, 33, 613-630.

Barrett, L. F., Lane, R., Sechrest, L., \& Schwartz, G. (2000). Sex differences in emotional awareness. Personality and Social Psychology Bulletin, 26, 1027-1035.

Bekker, M. H., \& van Mens-Verhulst, J. (2007). Anxiety disorders: Sex differences in prevalence, degree, and background, but genderneutral treatment. Gender Medicine, 4(Supp. B), S178-S193.

Buckner, R. L., Andrews-Hanna, J. R., \& Schacter, D. L. (2008). The brain's default network. Annals of the New York Academy of Sciences, 1124, 1-38.

Bush, G., Luu, P., \& Posner, M. I. (2000). Cognitive and emotional influences in anterior cingulate cortex. Trends in Cognitive Sciences, 4, 215-222.

Butler, R., Pan, H., Epstein, J., Protopopescu, X., Tuescher, O., Goldstein, M., \& Silbersweig, D. (2005). Fear-related activity in subgenual anterior cingulate differs between men and women. NeuroReport, 16, 1233-1236.

Canli, T., Desmond, J. E., Zhao, Z., \& Gabrieli, J. D. E. (2002). Sex differences in the neural basis of emotional memories. Proceedings of the National Academy of Sciences, 99, 1078910794. doi:10.1073/pnas.162356599

Chuah, L. Y. M., Dolcos, F., Chen, A. K., Zheng, H., Parimal, S., \& Chee, M. W. L. (2010). Sleep deprivation and interference by emotional distracters. Sleep, 33, 1305-1313.

Courtney, S. M., Petit, L., Maisog, J. M., Ungerleider, L. G., \& Haxby, J. V. (1998). An area specialized for spatial working memory in human frontal cortex. Science, 279, 1347-1351.

D'Argembeau, A., Stawarczyk, D., Majerus, S., Collette, F., Van der Linden, M., Feyers, D., \& Salmon, E. (2010). The neural basis of personal goal processing when envisioning future events. Journal of Cognitive Neuroscience, 22, 1701-1713.

D’Esposito, M. (2008). Working memory. In G. Goldenberg \& B. L. Miller (Eds.), Neuropsychology and behavioral neurology (Handbook of Clinical Neurology, Vol. 88, pp. 237-247). Edinburgh, U.K: Elsevier.

D'Esposito, M., Postle, B. R., \& Rypma, B. (2000). Prefrontal cortical contributions to working memory: Evidence from event-related fMRI studies. Experimental Brain Research, 133, 3-11. 
Denkova, E., Dolcos, S., \& Dolcos, F. (2012). Reliving emotional personal memories: Affective biases linked to personality and sex-related differences. Emotion, 12, 515-528. doi:10.1037/ a0026809

Denkova, E., Wong, G., Dolcos, S., Sung, K., Wang, L., Coupland, N., \& Dolcos, F. (2010). The impact of anxiety-inducing distraction on cognitive performance: A combined brain imaging and personality investigation. PLoS One, 5, e14150. doi:10.1371/ journal.pone.0014150

Diaz, M. T., He, G., Gadde, S., Bellion, C., Belger, A., Voyvodic, J. T., \& McCarthy, G. (2011). The influence of emotional distraction on verbal working memory: An fMRI investigation comparing individuals with schizophrenia and healthy adults. Journal of Psychiatric Research, 45, 1184-1193.

Dimberg, U., \& Lundquist, L.-O. (1990). Gender differences in facial reactions to facial expressions. Biological Psychology, 30, 151-159.

Dolcos, F., Diaz-Granados, P., Wang, L., \& McCarthy, G. (2008). Opposing influences of emotional and non-emotional distracters upon sustained prefrontal cortex activity during a delayedresponse working memory task. Neuropsychologia, 46, 326335. doi:10.1016/j.neuropsychologia.2007.07.010

Dolcos, F., Iordan, A. D., \& Dolcos, S. (2011). Neural correlates of emotioncognition interactions: A review of evidence from brain imaging investigations. Journal of Cognitive Psychology, 23, 669-694.

Dolcos, F., Kragel, P., Wang, L., \& McCarthy, G. (2006). Role of the inferior frontal cortex in coping with distracting emotions. NeuroReport, 17, 1591-1594.

Dolcos, F., LaBar, K. S., \& Cabeza, R. (2005). Remembering one year later: Role of the amygdala and the medial temporal lobe memory system in retrieving emotional memories. Proceeding of the National Academy of Sciences, 102, 2626-2631. doi:10.1073/ pnas.0409848102

Dolcos, F., \& McCarthy, G. (2006). Brain systems mediating cognitive interference by emotional distraction. Journal of Neuroscience, 26, 2072-2079.

Dolcos, F., Miller, B., Kragel, P., Jha, A., \& McCarthy, G. (2007). Regional brain differences in the effect of distraction during the delay interval of a working memory task. Brain Research, 1152, 171-181.

Domes, G., Schulze, L., Böttger, M., Grossmann, A., Hauenstein, K., Wirtz, P. H., \& Herpertz, S. C. (2010). The neural correlates of sex differences in emotional reactivity and emotion regulation. Human Brain Mapping, 31, 758-769. doi:10.1002/hbm.20903

Drevets, W. C., \& Raichle, M. E. (1998). Reciprocal suppression of regional cerebral blood flow during emotional versus higher cognitive processes: Implications for interactions between emotion and cognition. Cognition and Emotion, 12, 353-385.

Fisher, R. A. (1950). Statistical methods for research workers. London, U.K.: Oliver \& Boyd.

Goldstein, J. M., Seidman, L. J., Horton, N. J., Makris, N., Kennedy, D. N., Caviness, V. S., Jr., \& Tsuang, M. T. (2001). Normal sexual dimorphism of the adult human brain assessed by in vivo magnetic resonance imaging. Cerebral Cortex, 11, 490-497. doi:10.1093/cercor/11.6.490

Gotlib, I. H., Sivers, H., Gabrieli, J. D. E., Whitfield-Gabrieli, S., Goldin, P., Minor, K. L., \& Canli, T. (2005). Subgenual anterior cingulate activation to valenced emotional stimuli in major depression. NeuroReport, 16, 1731-1734.

Greicius, M. D., Krasnow, B., Reiss, A. L., \& Menon, V. (2003). Functional connectivity in the resting brain: A network analysis of the default mode hypothesis. Proceeding of the National Academy of Sciences, 100, 253-258.

Gur, R. C., Gunning-Dixon, F., Bilker, W. B., \& Gur, R. E. (2002). Sex differences in temporo-limbic and frontal brain volumes of healthy adults. Cerebral Cortex, 12, 998-1003.
Hamann, S., \& Canli, T. (2004). Individual differences in emotion processing. Current Opinion in Neurobiology, 14, 233-238.

Harrison, B. J., Pujol, J., Ortiz, H., Fornito, A., Pantelis, C., \& Yücel, M. (2008). Modulation of brain resting-state networks by sad mood induction. PLoS One, 3, e1794.

Hopfinger, J. B., Buonocore, M. H., \& Mangun, G. R. (2000). The neural mechanisms of top-down attentional control. Nature Neuroscience, 3, 284-291.

Kessler, R. C. (2003). Epidemiology of women and depression. Journal of Affective Disorders, 74, 5-13.

Koch, K., Pauly, K., Kellermann, T., Seiferth, N. Y., Reske, M., Backes, V., \& Habel, U. (2007). Gender differences in the cognitive control of emotion: An fMRI study. Neuropsychologia, 45, 2744-2754. doi:10.1016/j.neuropsychologia.2007.04.012

Kring, A. M., \& Gordon, A. H. (1998). Sex differences in emotion: Expression, experience, and physiology. Journal of Personality and Social Psychology, 74, 686-703.

Lang, P. J., Bradley, M. M., \& Cuthbert, B. N. (2008). International Affective Picture System (IAPS): Affective ratings of pictures and instruction manual (Technical Report No. A-8). Gainesville, FL: University of Florida, Center for Research in Psychophysiology.

Lang, P. J., Greenwald, M. K., Bradley, M. M., \& Hamm, A. O. (1993). Looking at pictures: Affective, facial, visceral, and behavioral reactions. Psychophysiology, 30, 261-273.

Levy, R., \& Goldman-Rakic, P. S. (2000). Segregation of working memory functions within the dorsolateral prefrontal cortex. Experimental Brain Research, 133, 23-32.

Lieberman, M. D., \& Cunningham, W. A. (2009). Type I and Type II error concerns in fMRI research: Re-balancing the scale. Social Cognitive and Affective Neuroscience, 4, 423-428. doi:10.1093/scan/nsp052

Macmillan, N. A., \& Creelman, C. D. (1991). Detection theory: A user's guide. New York, NY: Cambridge University Press.

Mak, A. K., Hu, Z. G., Zhang, J. X., Xiao, Z., \& Lee, T. M. (2009). Sex-related differences in neural activity during emotion regulation. Neuropsychologia, 47, 2900-2908.

Matud, M. R. (2004). Gender differences in stress and coping styles. Personality and Individual Differences, 37, 1401-1415.

Mayberg, H. S. (1997). Limbic-cortical dysregulation: A proposed model of depression. The Journal of Neuropsychiatry and Clinical Neurosciences, 9, 471-481.

McKiernan, K. A., Kaufman, J. N., Kucera-Thompson, J., \& Binder, J. R. (2003). A parametric manipulation of factors affecting task-induced deactivation in functional neuroimaging. Journal of Cognitive Neuroscience, 15, 394-408. doi:10.1162/089892903321593117

McRae, K., Ochsner, K. N., Mauss, I. B., Gabrieli, J. D. E., \& Gross, J. J. (2008). Gender differences in emotion regulation: An fMRI study of cognitive reappraisal. Group Processes \& Intergroup Relations, 11, 143-163. doi:10.1177/1368430207088035

Miller, E. K., \& Cohen, J. D. (2001). An integrative theory of prefrontal cortex function. Annual Review of Neuroscience, 24, 167-202. doi:10.1146/annurev.neuro.24.1.167

Mobbs, D., Marchant, J. L., Hassabis, D., Seymour, B., Tan, G., Gray, M., \& Frith, C. D. (2009). From threat to fear: The neural organization of defensive fear systems in humans. Journal of Neuroscience, 29, 12236-12243.

Morey, R. A., Dolcos, F., Petty, C. M., Cooper, D. A., Hayes, J. P., LaBar, K. S., \& McCarthy, G. (2009). The role of trauma-related distracters on neural systems for working memory and emotion processing in posttraumatic stress disorder. Journal of Psychiatric Research, 43, 809-817.

Nichols, T., Brett, M., Andersson, J., Wager, T., \& Poline, J.-B. (2005). Valid conjunction inference with the minimum statistic. NeuroImage, 25, 653-660.

Northoff, G., \& Bermpohl, F. (2004). Cortical midline structures and the self. Trends in Cognitive Sciences, 8, 102-107. 
Northoff, G., Heinzel, A., de Greck, M., Bermpohl, F., Dobrowolny, H., \& Panksepp, J. (2006). Self-referential processing in our brain-A meta-analysis of imaging studies on the self. NeuroImage, 31, 440 457. doi:10.1016/j.neuroimage. 2005.12 .002

Ochsner, K. N. (2000). Are affective events richly recollected or simply familiar? The experience and process of recognizing feelings past. Journal of Experimental Psychology. General, 129, 242-261. doi:10.1037/0096-3445.129.2.242

Ochsner, K. N., Knierim, K., Ludlow, D. H., Hanelin, J., Ramachandran, T., Glover, G., \& Mackey, S. C. (2004). Reflecting upon feelings: An fMRI study of neural systems supporting the attribution of emotion to self and other. Journal of Cognitive Neuroscience, 16, 1746-1772. doi:10.1162/ 0898929042947829

Orozco, S., \& Ehlers, C. L. (1998). Gender differences in electrophysiological responses to facial stimuli. Biological Psychiatry, 44, 281-289.

Phan, K. L., Taylor, S. F., Welsh, R. C., Ho, S. H., Britton, J. C., \& Liberzon, I. (2004). Neural correlates of individual ratings of emotional salience: A trial-related fMRI study. NeuroImage, 21, $768-780$.

Pitroda, S., Angstadt, M., McCloskey, M. S., Coccaro, E. F., \& Phan, K. L. (2008). Emotional experience modulates brain activity during fixation periods between tasks. Neuroscience Letters, 443, 72-76.

Raichle, M. E., MacLeod, A. M., Snyder, A. Z., Powers, W. J., Gusnard, D. A., \& Shulman, G. L. (2001). A default mode of brain function. Proceeding of the National Academy of Sciences, 98, 676-682. doi:10.1073/pnas.98.2.676

Schacter, D. L., Addis, D. R., \& Buckner, R. L. (2008). Episodic simulation of future events: Concepts, data, and applications. Annals of the New York Academy of Sciences, 1124, 39-60.

Seidlitz, L., \& Diener, E. (1998). Sex differences in the recall of affective experiences. Journal of Personality and Social Psychology, 74, 262-271.

Sharot, T., Delgado, M. R., \& Phelps, E. A. (2004). How emotion enhances the feeling of remembering. Nature Neuroscience, 7 , $1376-1380$.
Shields, S. A. (1991). Gender in the psychology of emotion: A selective research review. In K. T. Strongman (Ed.), International review of studies on emotion (pp. 227-245). New York, NY: Wiley.

Spielberger, C. D., Gorsuch, R. L., \& Lushene, R. E. (1970). Manual for the State-Trait Anxiety Inventory. Palo Alto, CA: Consulting Psychologists Press.

Svoboda, E., McKinnon, M. C., \& Levine, B. (2006). The functional neuroanatomy of autobiographical memory: A meta-analysis. Neuropsychologia, 44, 2189-2208.

Tanaka, S., Honda, M., \& Sadato, N. (2005). Modality-specific cognitive function of medial and lateral human Brodmann Area 6 . Journal of Neuroscience, 25, 496-501.

Thayer, J. F., Rossy, L. A., Ruiz-Padial, E., \& Johnsen, B. H. (2003). Gender differences in the relationship between emotional regulation and depressive symptoms. Cognitive Therapy and Research, 27, 349-364.

Tottenham, N., Borscheid, A., Ellertsen, K., Marcus, D. J., \& Nelson, C. A. (2002, April). Categorization of facial expressions in children and adults: Establishing a larger stimulus set. Paper presented at the Annual Meeting of the Cognitive Neuroscience Society, San Francisco, CA.

Wager, T. D., Phan, K. L., Liberzon, I., \& Taylor, S. F. (2003). Valence, gender, and lateralization of functional brain anatomy in emotion: A meta-analysis of findings from neuroimaging. NeuroImage, 19, 513-531.

Watson, D., Clark, L. A., \& Tellegen, A. (1988). Development and validation of brief measures of positive and negative affect: The PANAS scales. Journal of Personality and Social Psychology, 54, 1063-1070. doi:10.1037/0022-3514.54.6.1063

Weissman-Fogel, I., Moayedi, M., Taylor, K. S., Pope, G., \& Davis, K. D. (2010). Cognitive and default-mode resting state networks: Do male and female brains "rest" differently? Human Brain Mapping, 31, 1713-1726. doi:10.1002/hbm.20968

Wiebking, C., de Greck, M., Duncan, N. W., Heinzel, A., Tempelmann, C., \& Northoff, G. (2011). Are emotions associated with activity during rest or interoception? An exploratory fMRI study in healthy subjects. Neuroscience Letters, 491, 87-92. 